\title{
直接二氟甲硫基化与一氟甲硫基化反应研究进展
}

\author{
间强蒋绿齐*易文斌* \\ (南京理工大学化工学院 南京 210094)
}

\begin{abstract}
摘要 含有二氟甲硫基基团 $\left(\mathrm{SCF}_{2} \mathrm{H}\right)$ 与含有一氟甲硫基基团 $\left(\mathrm{SCFH}_{2}\right)$ 的有机化合物具有独特的物理和化学性质，在医药 及农药等领域具有潜在的应用价值. 这两类化合物的传统制备方法是颈基底物的二氟甲基化与一氟甲基化，但含統基 底物本身种类有限，极大地限制了该类化合物的应用与开发，因此开发新的直接二氟/一氟甲硫基化方法和开拓新型直 接二氟/一氟甲硫基化试剂具有重要的意义. 综述了直接二氟甲硫基化反应和一氟甲硫基化反应的最新研究进展, 并对 反应的相关机理进行了论述.
\end{abstract}

关键词 二氟甲硫基化; 一氟甲硫基化; 直接法

\section{Recent Progress on Direct Difluoromethylthiolation and Monofluoromethylthiolation}

\author{
Yan, Qiang Jiang, Lüqi* Yi, Wenbin* \\ (Chemical Engineering College, Nanjing University of Science and Technology, Nanjing 210094)
}

\begin{abstract}
Compounds bearing difluoromethylthio $\left(\mathrm{SCF}_{2} \mathrm{H}\right)$ and monofluoromethylthio $\left(\mathrm{SCFH}_{2}\right)$ groups are potentially important targets in the pharmaceutical and agrochemical fields due to their unique physical and chemical properties. The traditional methods of synthesizing these two kinds of compounds are difluoromethylation and monofluoromethylation of sulfhydryl substrates. However, the limitation of sulfhydryl substrates also limited the application and development of such compounds. Thus, it is still highly desirable to develop new methods for difluoromethylthiolation and monofluoromethylthiolation as well as new types of difluoromethylthiolation and monofluoromethylthiolation reagents. The recent development of direct difluoromethylthiolation and monofluoromethylthiolation reactions is summarized, and the related mechanism are also discussed.

Keywords difluoromethylthiolation; monofluoromethylthiolation; direct method
\end{abstract}

含有一氟甲硫基 $\left(\mathrm{SCFH}_{2}\right)$ 、二氟甲硫基 $\left(\mathrm{SCF}_{2} \mathrm{H}\right)$ 的有 机分子与含有全氟烷硫基官能团的活性分子相比, 有着 完全不同的理化性质. 这两类分子与氟原子相连的碳原 子上分别含有一个和两个氢原子, 在氟原子作用下, 这 些氢具有一定的酸性，容易产生分子内以及分子间氢键 等相互作用 ${ }^{[1,2]}$, 可能会增加该分子在生物体内的代谢 稳定性. 此外, $\mathrm{SCF}_{2} \mathrm{H}$ 和 $\mathrm{SCFH}_{2}$ 具有介于 $\mathrm{SCF}_{3}$ 与 $\mathrm{SCH}_{3}$ 之间中等的 Hansch 亲脂系数, 也为药物的脂溶性调节 提供了一种可能.

尽管目前含有 $\mathrm{SCF}_{2} \mathrm{H}$ 与 $\mathrm{SCFH}_{2}$ 的药物还不是很多, 但其独特的性质已经引起化学及生物学工作者的注意,
部分含 $\mathrm{SCF}_{2} \mathrm{H}$ 和 $\mathrm{SCFH}_{2}$ 的活性分子已经成功地进入了 相应的应用领域. 图 1 是一些含有 $\mathrm{SCF}_{2} \mathrm{H}$ 及 $\mathrm{SCFH}_{2}$ 的 农药和医药活性分子. 化合物 $\mathbf{I}^{[3]}$ 是吡唑类杀虫剂氟虫 腈的同系物, 其降低了氟虫腈对蜜蜂以及水生动物的毒 性, 成为一种新型的广谱杀虫剂; 化合物 II 是一个除草 活性分子 ${ }^{[4]}$; 化合物 $\mathbf{I I I}^{[5]}$ 是抗心绞痛药物弥新平的同类 活性分子; 化合物 $\mathbf{I} \mathbf{V}^{[6,7]}$ 和 $\mathbf{V}^{[8]}$ 是分别可用作抗生素和 抵抗人类免疫缺陷病毒(HIV-1)的活性分子; 化合物 VI 则是一个潜在的杀菌活性分子 ${ }^{[9]}$; 化合物 $\mathbf{V I I} \sim \mathbf{X}$ 是消

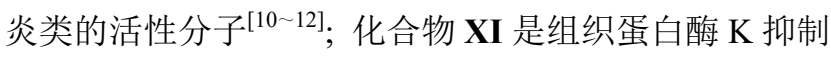
剂. 由于 $\mathrm{SCF}_{2} \mathrm{H}$ 和 $\mathrm{SCFH}_{2}$ 潜在的应用价值较大,

\footnotetext{
* Corresponding authors. E-mail: lvqi.jiang@njust.edu.cn; yiwb@njust.edu.cn

Received July 20, 2019; revised August 29, 2019; published online September 13, 2019.

Project supported by the National Natural Science Foundation of China (No. 21476116), the Fundamental Research Funds for the Central Universities (No. 30918011314), the Natural Science Foundation of Jiangsu Province (No. BK20141394) and the Qing Lan and Six Talent Peaks in Jiangsu Province.

国家自然科学基金(No. 21476116)、中央高校基本科研业务费专项资金(No. 30918011314)、江苏省自然科学基金(No. BK20141394)和江苏省青蓝计划 和六大高峰人才资助项目.
} 
<smiles>N#Cc1nn(-c2c(Cl)cc(C(F)(F)F)cc2Cl)c(NCc2ccccn2)c1[OH+]</smiles>

I (Pyriprole)<smiles>CC(C)Nc1nc(NC(C)C)nc([Se-]C(F)(F)F)n1</smiles>

II (SSH-108)<smiles>CCOc1ccccc1-c1c(C(=O)OC)c(C)nc(C)c1C(=O)OC</smiles>

III (Nifedipin analogue)<smiles>CC1(NC(=O)CSC(F)(F)F)C(=O)C2C(C(N)=O)=C(CSc3nnnn3CCO)CCC21O</smiles>

IV (Antibiotic)<smiles>Cc1ccnc(SC(F)(F)c2nc3ccccc3o2)n1</smiles>

V (Against HIV-1)<smiles>[R]Nc1c([N+](=O)[O-])cc([SeH])cc1[N+](=O)[O-]</smiles>

VI (Potential fungcides)

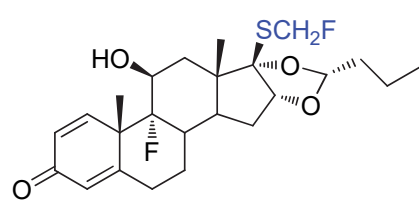

VII (Androstanes)

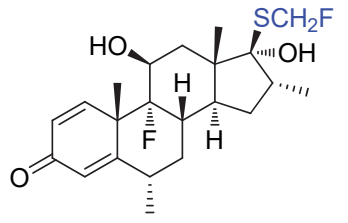

VIII (Androstanes)

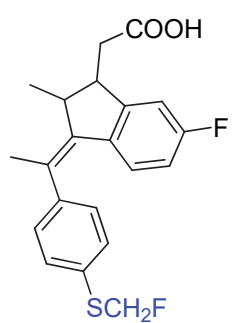

IX (Sulindac)

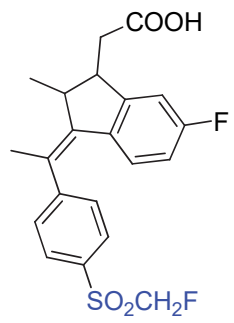

X (Sulindac)<smiles>N#CCNC(=O)[C@H]1CC(F)(F)CC[C@H]1c1ccccc1-c1ccc(CF)cc1</smiles>

XI (Cat K inhibitior)

图 1 含有 $\mathrm{SCF}_{2} \mathrm{H}$ 和 $\mathrm{SCFH}_{2}$ 基团的活性分子

Figure $1 \mathrm{SCF}_{2} \mathrm{H} / \mathrm{SCFH}_{2}$ containing bioactive compounds

开发出更好的合成方法和合成试剂变得越来越重要. 本 文就近年来不含颈基底物的直接二氟甲硫基化和直接 一氟甲硫基化反应研究进展进行概述.

\section{1 二氟甲硫基化反应}

二氟甲硫基引入分子的方法分为直接法和间接法 两类. 早期合成该类化合物的方法需要先制备含有颈基 的底物，再使用不同的二氟甲基化试剂对含硫底物的硫 原子进行二氟甲基化, 称为间接法(Scheme 1). 该方法 具有反应条件简单及氟化试剂经济等特点. 但由于含

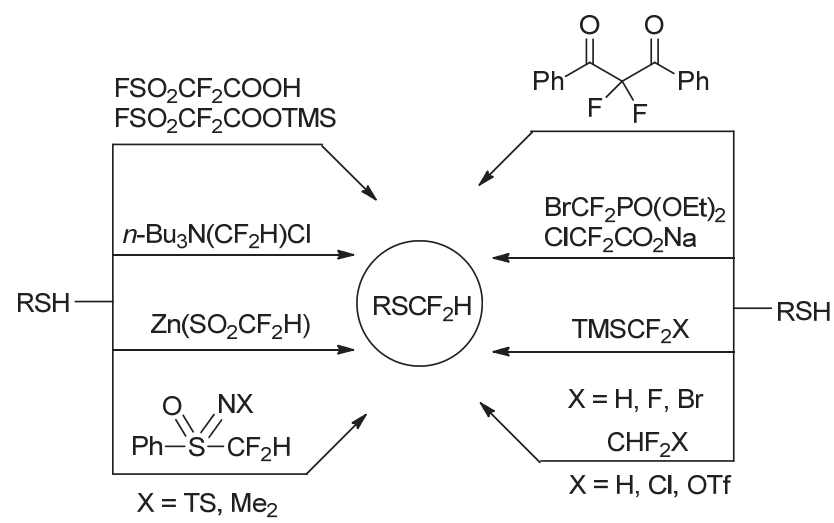

图式 1 间接引入二氟甲硫基的方法

Scheme 1 Indirect methods for difluoromethylthiolation
颈基底物本身种类有限, 复杂底物的硫醇本身制备也比 较困难，极大地限制了含 $\mathrm{SCF}_{2} \mathrm{H}$ 官能团的活性分子在 工业领域的应用与开发 ${ }^{[13 \sim 31]}$.

不含硫底物的直接二氟甲硫基化方法即直接法，是 含有 $\mathrm{SCF}_{2} \mathrm{H}$ 官能团分子的另一个重要的合成方法，也 是本文综述的重点. 这类方法起步较晚, 但对反应底物 要求较低, 合成过程简洁高效. 从 2015 年开始, 不含硫 底物的直接二氟甲硫基化反应的报道陆续出现，成为有 机氟化学领域的研究热点. 根据二氟甲硫基来源, 将该 方法分为亲核、亲电以及自由基三个反应类型，并逐一 进行论述.

\section{1 亲电二氟甲硫基化}

2015 年, 沈其龙和吕龙课题组 ${ }^{[32]}$ 用邻苯二甲酰亚 胺和课题组自研试剂 $(\mathrm{SIPr}) \mathrm{Ag}\left(\mathrm{SCF}_{2} \mathrm{H}\right)$ 为主要原料, 共 同开发了第一个直接亲电二氟甲硫基化试剂(Eq. 1)，该 试剂在合成上具有重要的应用价值.<smiles>O=C1c2ccccc2C(=O)N1Cl</smiles>

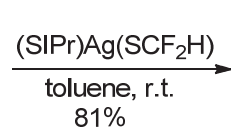<smiles>O=C1c2ccccc2C(=O)N1C(F)(F)F</smiles>

通过对该试剂的深入研究，作者发现其适用范围非 常广泛，可以与很多种类底物发生亲电反应. 在 $\mathrm{TMSCl}$ 
催化下, $\mathrm{SCF}_{2} \mathrm{H}$ 可以被直接引入到吲哚、吡咯或富电子 苯环中; 在金属 $\mathrm{Cu}(\mathrm{I})$ 催化下, $\mathrm{SCF}_{2} \mathrm{H}$ 可以直接与苯硼酸 和末端炔烃反应; 在弱碱 $\mathrm{K}_{2} \mathrm{CO}_{3}$ 条件下, 该试剂则可以 平稳地与 $\beta$ 二羰基化合物的活泼氢发生取代反应; 在无 其他辅助试剂的条件下, 该试剂可以直接取代胺氢和颈 基氢生成 $\mathrm{NSCF}_{2} \mathrm{H}$ 和 $\mathrm{SSCF}_{2} \mathrm{H}$, 收率较高(Scheme 2).

$\mathrm{PhthNSCF}_{2} \mathrm{H}$ 作为第一个高效的亲电二氟甲硫基化 试剂有着极高的应用价值, 但其制备中用到的(SIPr)Ag$\left(\mathrm{SCF}_{2} \mathrm{H}\right)$ 价格昂贵, 制备过程复杂, 极大地限制了使用. 为此, 沈其龙课题组 ${ }^{[33]}$ 两年后提出了新的制备方法 (Eq. 2). 新方法可以达到克级规模的生产, 大大降低了 试剂合成的难度与成本. 同时, 也将该试剂成功地应用 于一系列有工业化潜力的含有 $\mathrm{SCF}_{2} \mathrm{H}$ 活性分子的放量 合成中.

2013 年, Shibata 课题组 ${ }^{[34]}$ 开发出了一种基于高价 碘叶立德类型的亲电三氟甲硫基化试剂, 并在此工作基 础上于 2016 年制得了一类结构新颖且高效的高碘二氟 甲硫基化试剂 ${ }^{[35]}$. 其制备过程是先用二氟甲基亚磺酸 钠与溴代苯乙酮反应, 得到二氟磺酰基苯乙酮, 随后在 氢氧化钾的作用下与等物质的量的醋酸碘苯缩合, 从而 制得产物 $\mathbf{c}$ (Scheme 3).

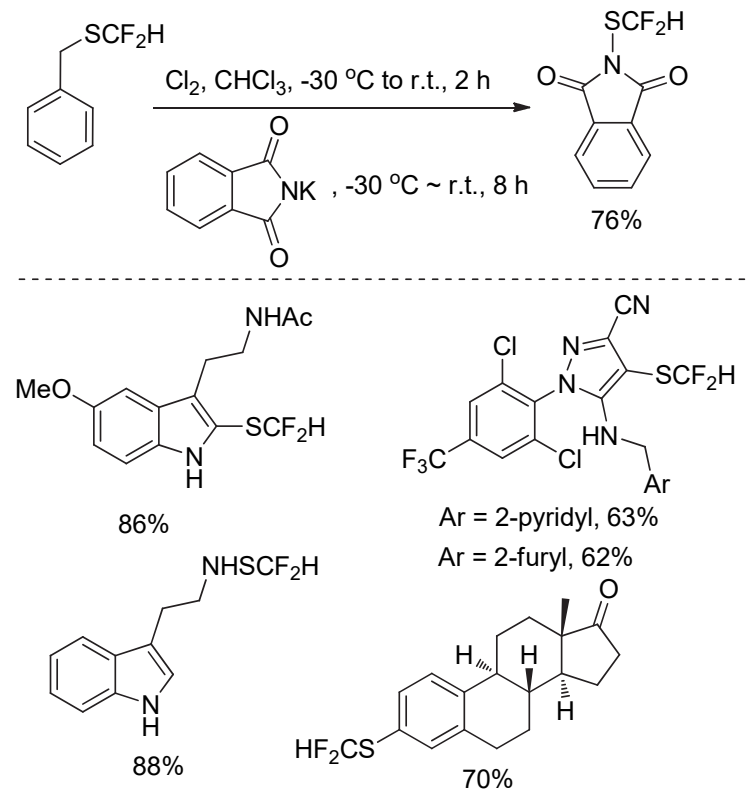

虽然化合物 $\mathbf{c}$ 与高碘三氟甲硫基试剂结构相似，但 该试剂在直接套用三氟甲硫基化反应条件 $[\mathrm{Cu}(\mathrm{I})$ 催化 $]$ 时，活性较低. 鉴于此，作者对反应条件进行了一系列 探索, 发现在 $\mathrm{CuBr}$ 催化下, 以二氧六环作溶剂, 该试剂 在室温下就可以与 $\beta$ 二羰基化合物及富电子的芳环(如
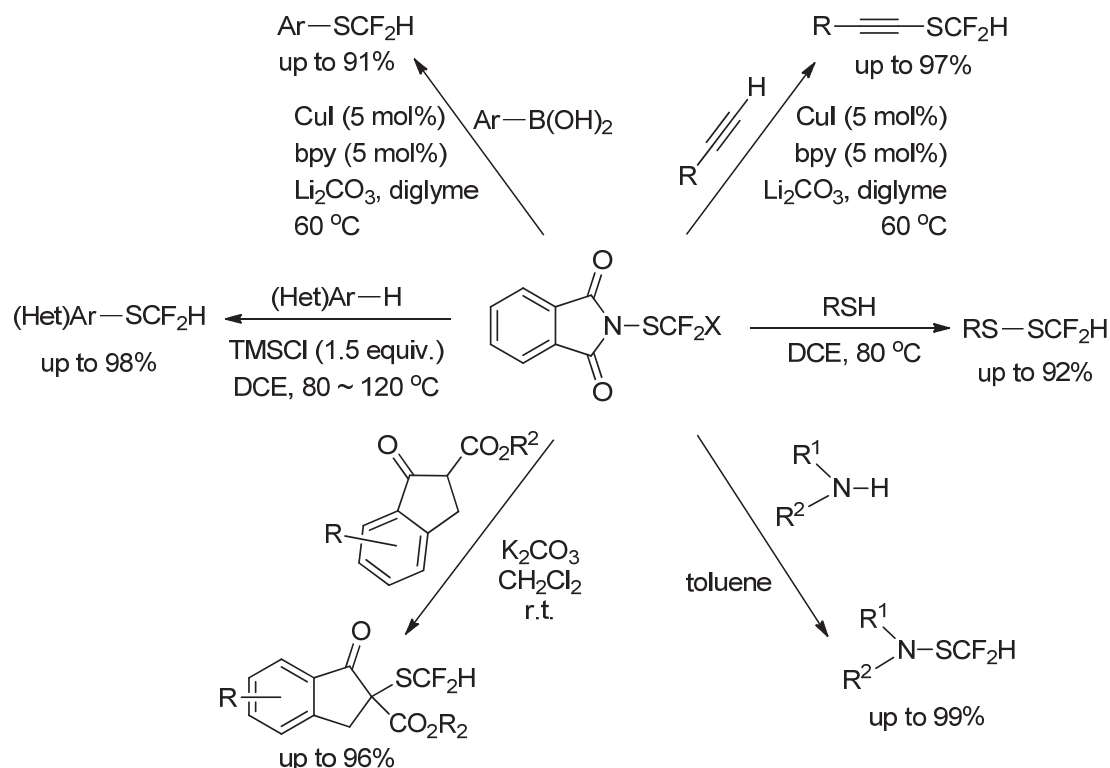

图式 2 Shen 试剂参与的亲电二氟甲硫基化反应

Scheme 2 Electrophilic difluoromethylthiolation using Shen's reagent<smiles>[R]c1ccc(C(=O)CBr)cc1</smiles>

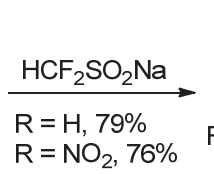

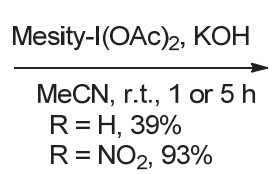

$\mathrm{R}=\mathrm{NO}_{2}, 93 \%$<smiles>[R]c1ccc(C(=O)C(=Ic2c(C)cc(C)cc2C(F)(F)F)S(=O)(=O)OCC)cc1</smiles>

图式 3 Shibata 试剂的合成

Scheme 3 Preparation of Shibata reagent 
吲哚和吡咯等)底物发生反应, 收率中等 ${ }^{[35]}$. 此外, 作者 还进一步拓展了该试剂在手性化合物合成方面的应用. 以 $\mathrm{CuBr}$ 为催化剂, 用含有手性萗胺的烯胺化合物与该 试剂在甲苯中室温反应, 可以中等的收率得到含有 $\mathrm{SCF}_{2} \mathrm{H}$ 的手性化合物, ee 值最高可达 $93 \%$ (Scheme 4) ${ }^{[36]}$.

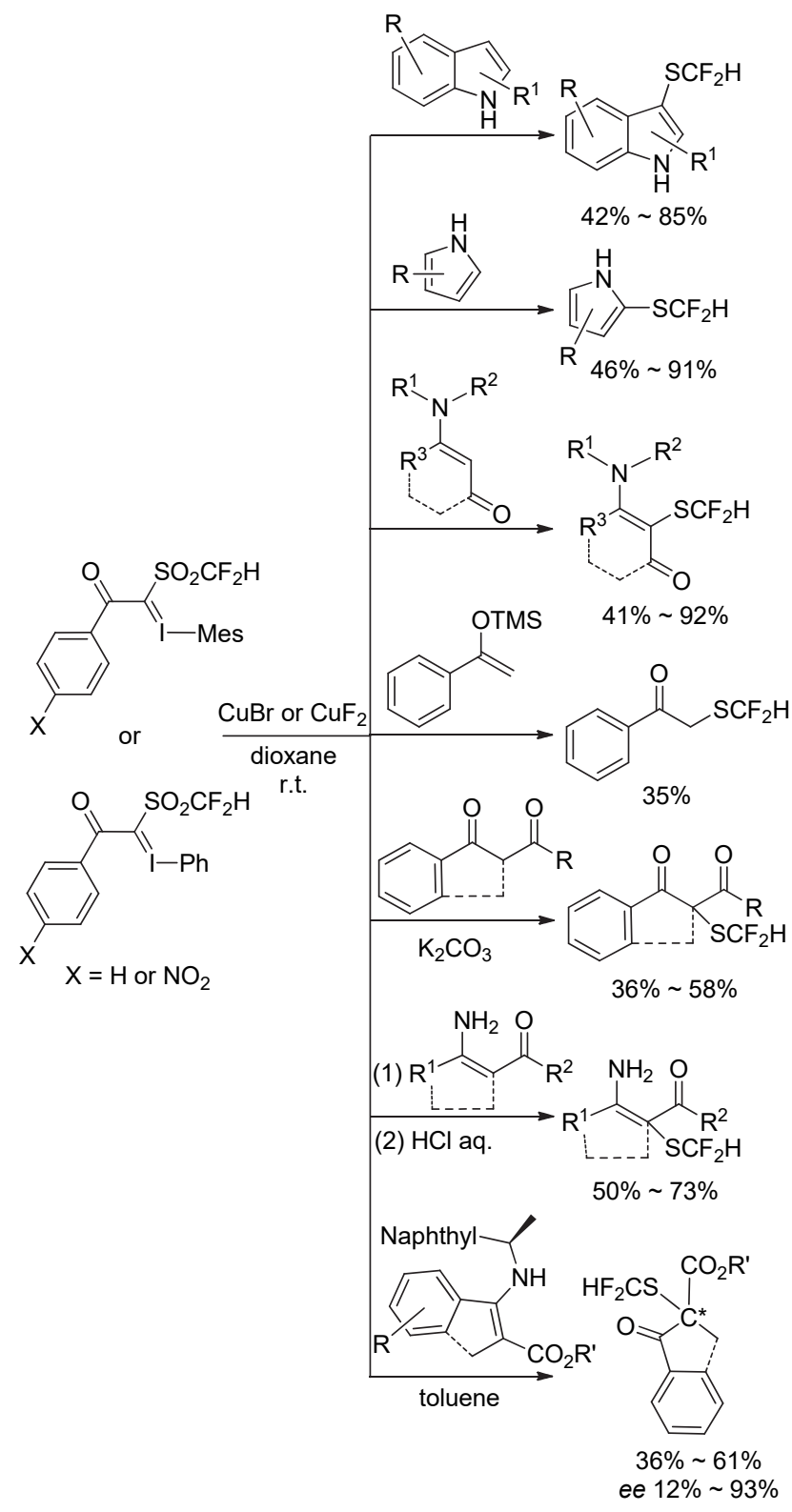

图式 4 Shibata 试剂的反应

Scheme 4 Reactions of Shibata reagent

作者推测了该反应可能的反应机理. 在 $\mathrm{CuBr}$ 催化 下，试剂分子内高价碘片段离去，形成卡宾中间体. 之 后邻位硫 $(\mathrm{S})$ 上的两个氧 $(\mathrm{O})$ 发生分子内重排, 形成含有 $\mathrm{SCF}_{2} \mathrm{H}$ 片段的活性酯. 最后对富电子底物进行取代或加 成反应，得到了最终二氟甲硫基化产物(Scheme 5).

在开发以上新试剂的同时, 科研工作者也通过现有 廉价易得的化工原料, 寻求更好的直接二氟甲硫基化方

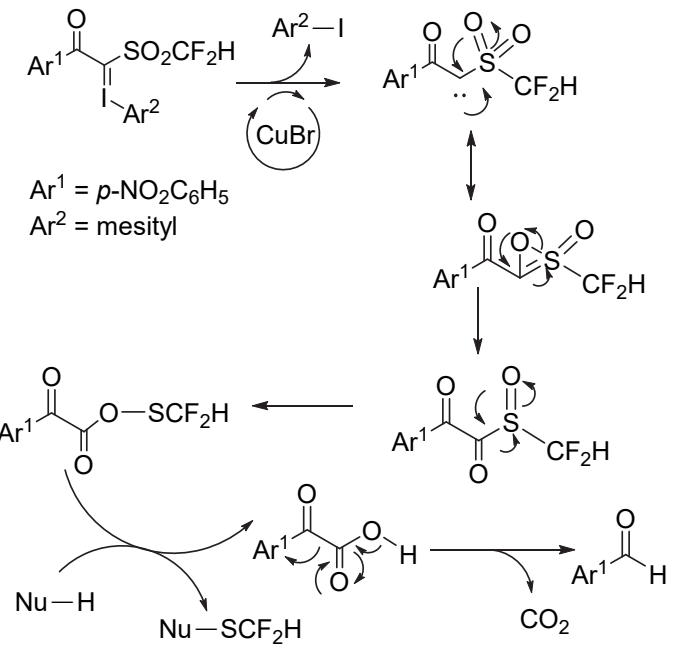

图式 5 Shibata 试剂参与的二氟甲硫基化反应机理推测 Scheme 5 Proposed mechanism for difluoromethylthiolation using Shibata reagent

法. 二氟甲基磺酰氯 $\mathrm{HCF}_{2} \mathrm{SO}_{2} \mathrm{Cl}$ 是一种易得的有机试 剂, 2016 年我们课题 ${ }^{[37]}$ 组首先报道了 $\mathrm{HCF}_{2} \mathrm{SO}_{2} \mathrm{Cl}$ 在还原 剂亚磷酸二乙酯 $(\mathrm{EtO}){ }_{2} \mathrm{P}(\mathrm{O}) \mathrm{H}$ 存在的条件下, 发生亲电 取代反应，直接得到二氟甲硫基产物. 其研究底物包括 吡咯和吲哚等富电子芳环，收率中等(Scheme 6).

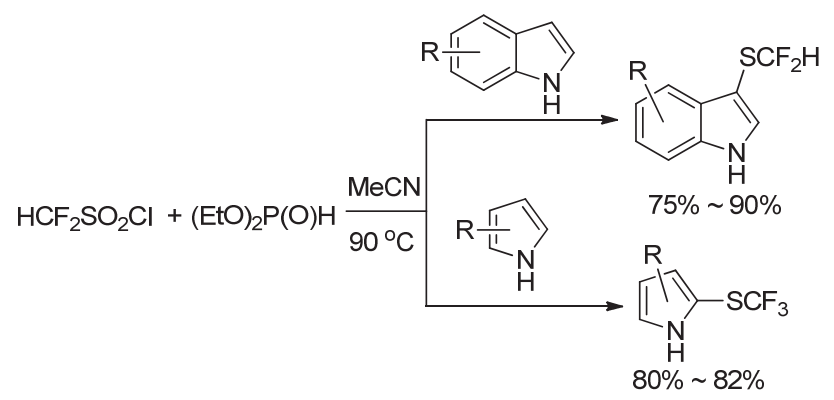

图式 $6 \mathrm{HCF}_{2} \mathrm{SO}_{2} \mathrm{Cl}$ 与 $(\mathrm{EtO})_{2} \mathrm{P}(\mathrm{O}) \mathrm{H}$ 参与的亲电二氟甲硫基化 反应

Scheme 6 Electrophilic difluoromethylthiolation of $\mathrm{HCF}_{2} \mathrm{SO}_{2} \mathrm{Cl}$ with $(\mathrm{EtO}){ }_{2} \mathrm{P}(\mathrm{O}) \mathrm{H}$

2017 年, 芦逵课题组进一步探索了 $\mathrm{HCF}_{2} \mathrm{SO}_{2} \mathrm{Cl}$ 作 为氟源的直接二氟甲硫基化反应条件. 在催化量的四丁 基碘化铵和三苯基膦的存在下，以吲哚、富电子五元杂 环 ${ }^{[38]}$ 和硫酚等 ${ }^{[39]}$ 为底物, 反应均可以获得较高收率的 产物(Scheme 7).

作者推测反应经历了以下过程, 首先 $\mathrm{CF}_{2} \mathrm{HSO}_{2} \mathrm{Cl}$ 在三苯基膦 $\left(\mathrm{PPh}_{3}\right)$ 与四丁基碘化铵的作用下得到二氟甲 基连二硫醚 $\left(\mathrm{CF}_{2} \mathrm{HSSCF}_{2} \mathrm{H}\right)$, 生成单质碘的同时 $\mathrm{PPh}_{3}$ 转 化为三苯氧膦 $\left(\mathrm{Ph}_{3} \mathrm{PO}\right)$. 紧接着单质碘与 $\mathrm{PPh}_{3}$ 生成碘化 三苯基膦 $\left(\mathrm{Ph}_{3} \mathrm{P}^{+} \mathrm{I}^{-}\right)$. 随后 $\mathrm{CF}_{2} \mathrm{HSO}_{2} \mathrm{Cl}$ 被 $\mathrm{Ph}_{3} \mathrm{P}^{+} \mathrm{I}^{-}$两次还 原，脱掉两个氧原子得到二氟甲基氯化硫 $\left(\mathrm{CF}_{2} \mathrm{HSCl}\right)$. 最后 $\mathrm{CF}_{2} \mathrm{HSCl}$ 与吲哚发生反应得到二氟甲硫基吲哚 
(Scheme 8).

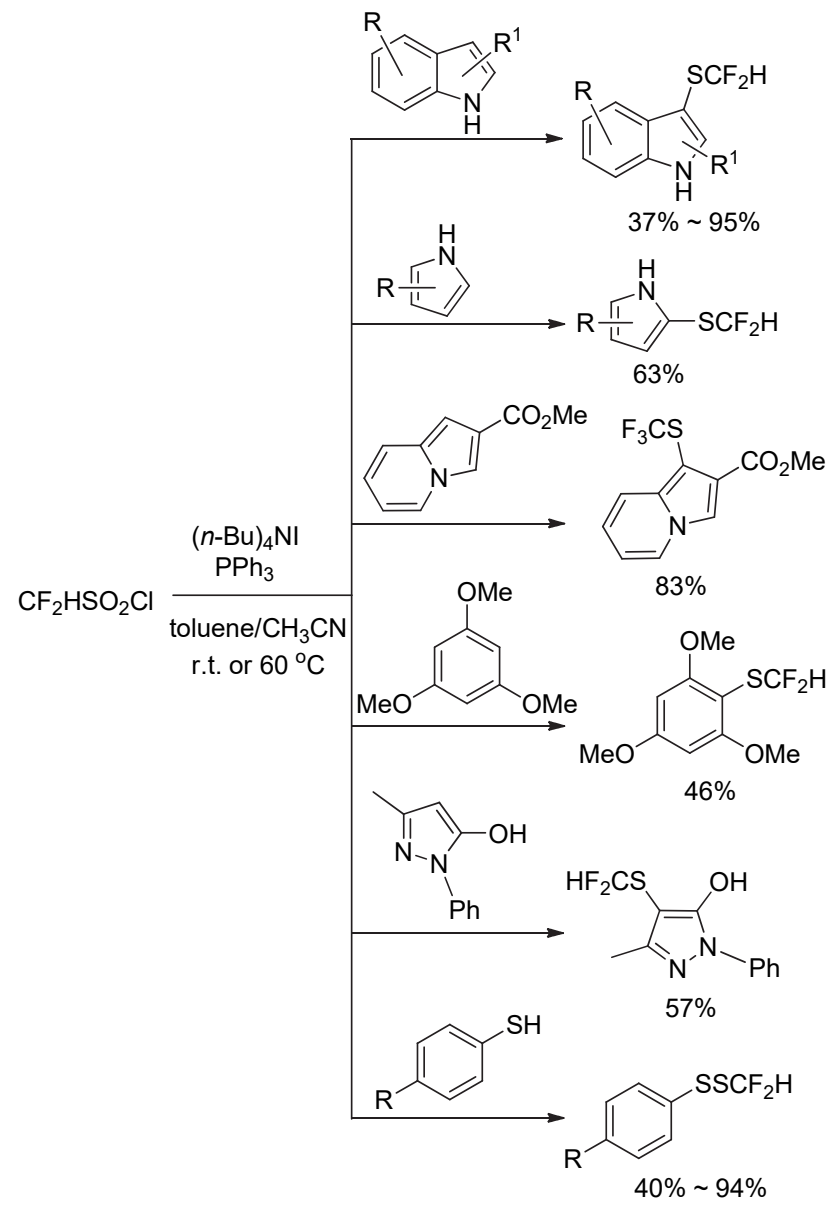

图式 $7 \mathrm{HCF}_{2} \mathrm{SO}_{2} \mathrm{Cl}$ 与 $\mathrm{PPh}_{3}$ 参与的亲电二氟甲硫基化反应 Scheme 7 Electrophilic difluoromethylthiolation of $\mathrm{HCF}_{2} \mathrm{SO}_{2} \mathrm{Cl}$ with $\mathrm{PPh}_{3}$

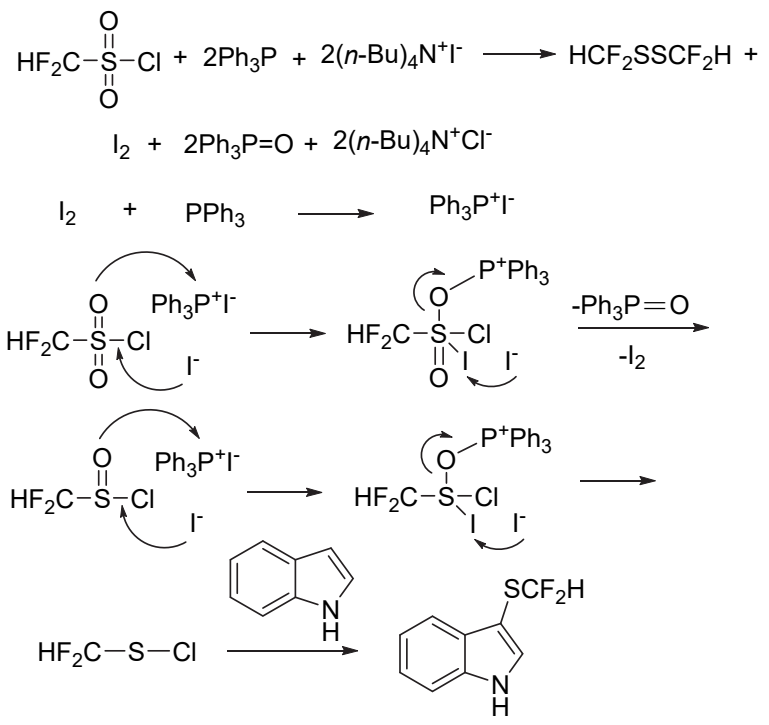

图式 $8 \mathrm{CF}_{2} \mathrm{HSO}_{2} \mathrm{Cl}$ 在 $\mathrm{PPh}_{3}$ 作用下的二氟甲硫基化反应机理 Scheme 8 Proposed mechanism for difluoromethylthiolation using $\mathrm{CF}_{2} \mathrm{HSO}_{2} \mathrm{Cl}$ and $\mathrm{PPh}_{3}$
除了亲电取代反应以外, 我们课题组 ${ }^{[40]}$ 于 2018 年 还报道了 $\mathrm{CF}_{2} \mathrm{HSO}_{2} \mathrm{Cl}$ 与炔烃和烯烃的亲电加成反应. 在三苯基膦的作用下，二氟甲基磺酰氯发生还原反应并 与不饱和双键或参键加成，得到取代环硫乙烷中间体， 同时氯离子对环硫乙烷进行开环加成, 得到同时含有氯 与二氟甲硫基双官能团的产物，收率良好(Eq. 3).

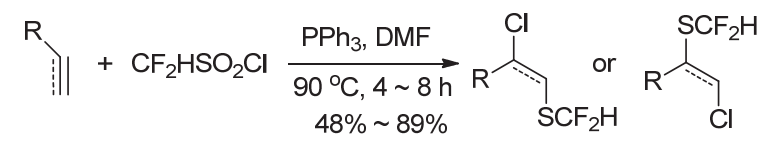

二氟甲基亚磺酰氯 $\left(\mathrm{CF}_{2} \mathrm{HSOCl}\right)$ 与二氟甲基磺酰氯 $\left(\mathrm{CF}_{2} \mathrm{HSO}_{2} \mathrm{Cl}\right)$ 组成相似，均含有 $\mathrm{CF}_{2} \mathrm{H}$ 与 $\mathrm{S}$ 原子，而且 $\mathrm{CF}_{2} \mathrm{HSOCl}$ 的硫原子价态比 $\mathrm{CF}_{2} \mathrm{HSO}_{2} \mathrm{Cl}$ 更低, 更容易还 原得到 $\mathrm{CF}_{2} \mathrm{HS}$ 官能团. 若将其作为二氟甲硫基化试剂, 具有更高的原子经济性，但该类反应的报道目前非常 少. 2018 年, 我们课题组 ${ }^{[1]}$ 在研究三氟甲基亚磺酰氯 $\left(\mathrm{CF}_{3} \mathrm{SOCl}\right)$ 的亲电取代时发现, 亲电底物与 $\mathrm{CF}_{3} \mathrm{SOCl}$ 发 生酰基化反应生成亚砜底物后, 过量 $\mathrm{CF}_{3} \mathrm{SOCl}$ 会继续与 亚砜发生还原反应，并最终脱氧得到三氟甲硫基化合 物。该策略应用于 $\mathrm{CF}_{2} \mathrm{HSOCl}$ 同样适用。过量的 $\mathrm{CF}_{2} \mathrm{HSOCl}$ 会将二氟甲基亚砜底物还原，一步得到二氟 甲硫基化合物，收率良好(Scheme 9).

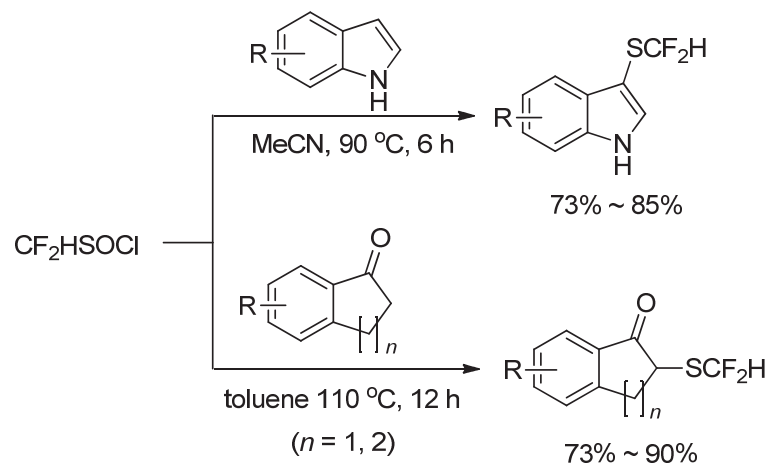

图式 9 二氟甲基亚磺酰氯自身还原的二氟甲硫基化反应

Scheme 9 Electrophilic difluoromethylthiolation of $\mathrm{HCF}_{2} \mathrm{SOCl}$ by self-reducing

与 $\mathrm{CF}_{2} \mathrm{HSO}_{2} \mathrm{Cl}$ 和 $\mathrm{CF}_{2} \mathrm{HSOCl}$ 相比，二氟甲基亚磺酸 钠 $\left(\mathrm{CF}_{2} \mathrm{HSO}_{2} \mathrm{Na}\right)$ 具有低毒稳定的特点, 是另一种理想的 二氟甲硫基化试剂. 2017 年, 我们课题组 ${ }^{[42]}$ 使用二氟甲 基亚磺酸钠作为氟化试剂, 实现了一系列化合物的亲电 二氟甲硫基化反应。该试剂通过与亚磷酸二乙酯以及 $\mathrm{TMSCl}$ 共同作用, 可以在富电子芳环和羰基 $\alpha$ 位上发生 二氟甲硫基化反应(Scheme 10).

我们课题组在反应条件探索的基础上，对反应进行 核磁跟踪，进一步推测了可能的反应机理. $\mathrm{CF}_{2} \mathrm{HSO}_{2} \mathrm{Na}$ 在路易斯酸作用下首先脱去一个氧原子，得到二氟甲基 


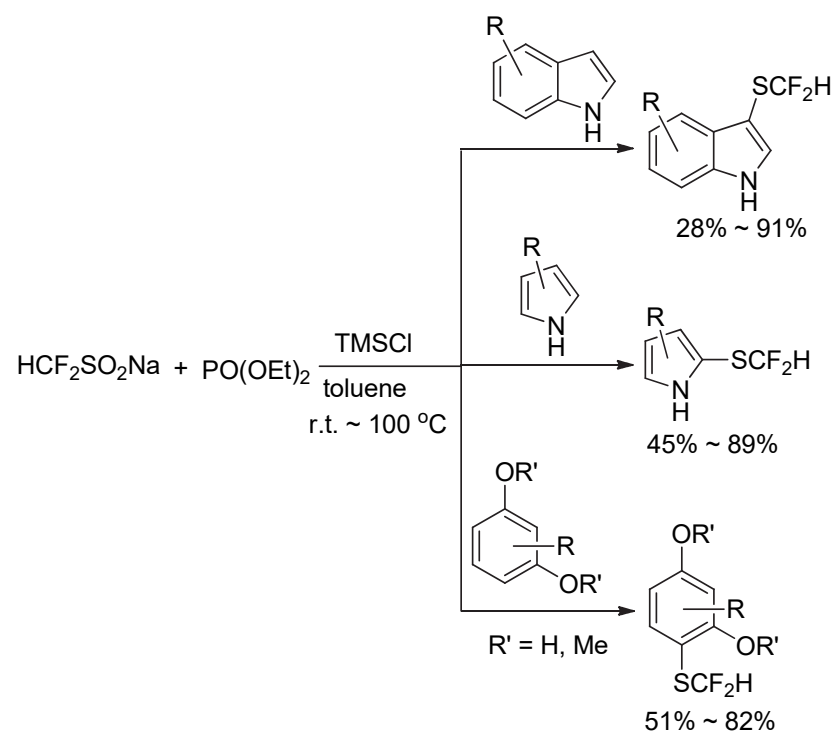

图式 $10 \quad \mathrm{HCF}_{2} \mathrm{SO}_{2} \mathrm{Na}$ 与 $(\mathrm{EtO})_{2} \mathrm{P}(\mathrm{O}) \mathrm{H}$ 参与的亲电二氟甲硫基 化反应

Scheme 10 Electrophilic difluoromethylthiolation of $\mathrm{HCF}_{2} \mathrm{SO}_{2} \mathrm{Na}$ with $(\mathrm{EtO})_{2} \mathrm{P}(\mathrm{O}) \mathrm{H}$

亚砜，随后重排为二氟甲硫基醇，进而在酸性条件下与 富电子底物进行亲电取代反应(Scheme 11).

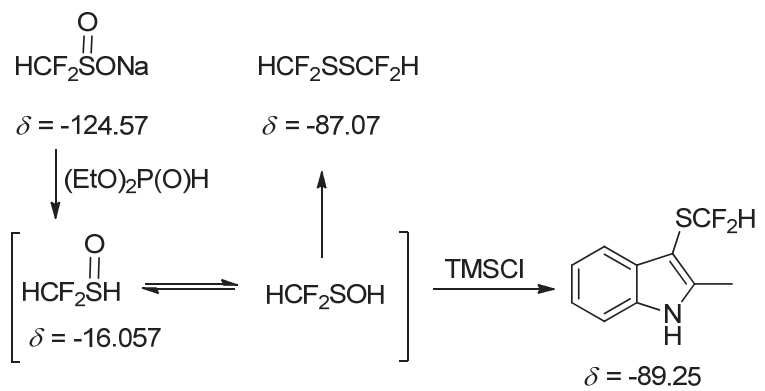

图式 $11 \mathrm{HCF}_{2} \mathrm{SO}_{2} \mathrm{Na}$ 在 $(\mathrm{EtO})_{2} \mathrm{P}(\mathrm{O}) \mathrm{H}$ 作用下的二氟甲硫基化 反应机理

Scheme 11 Proposed mechanism for difluoromethylthiolation using $\mathrm{HCF}_{2} \mathrm{SO}_{2} \mathrm{Na}$ and $(\mathrm{EtO})_{2} \mathrm{P}(\mathrm{O}) \mathrm{H}$

Shibata 课题组 ${ }^{[43]}$ 也几乎同时报道了二氟甲基亚磺 酸钠 $\left(\mathrm{HCF}_{2} \mathrm{SO}_{2} \mathrm{Na}\right)$ 参与的直接二氟甲硫基化反应.不同 的是，该反应使用了二苯基氯化磷 $\left(\mathrm{Ph}_{2} \mathrm{PCl}\right)$ 作为还原剂, 并且采用了两步法, 先让二苯基氯化磷与 $\mathrm{HCF}_{2} \mathrm{SO}_{2} \mathrm{Na}$ 反应, 得到活泼中间态后, 再加入亲电底物 (Scheme 12). 该反应条件收率较高, 底物使用范围也比较广.

\section{2 亲核二氟甲硫基化}

众所周知，三氟甲硫基金属盐是一类很常见的亲核 三氟甲硫基化试剂 ${ }^{[44,45]}$. 据此推断二氟甲硫基金属盐可 能也是一类重要的二氟甲硫基化试剂. 而与三氟甲硫基 阴离子能稳定地与金属成盐的性质不同，简单的二氟甲

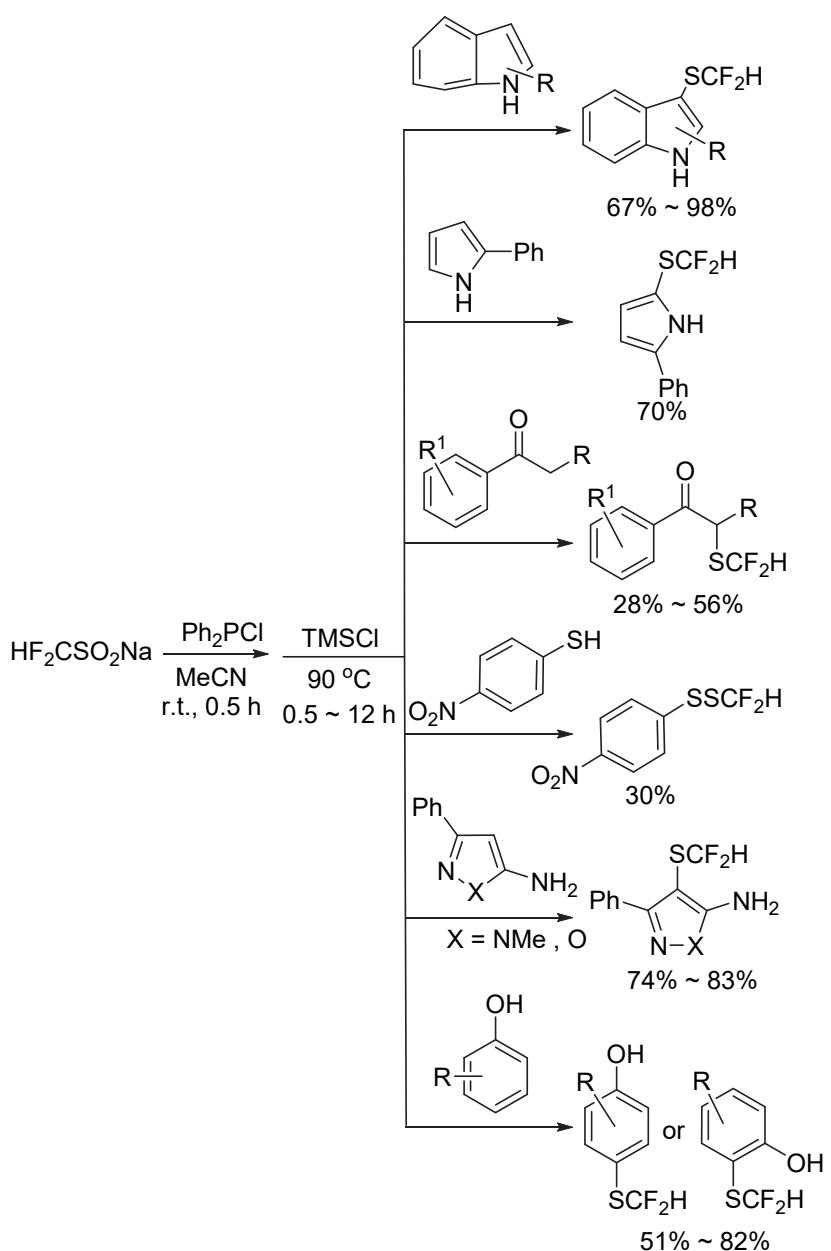

图式 $12 \mathrm{HCF}_{2} \mathrm{SO}_{2} \mathrm{Na}$ 与 $\mathrm{Ph}_{2} \mathrm{PCl}$ 参与的亲电二氟甲硫基化反应 Scheme 12 Electrophilic difluoromethylthiolation of $\mathrm{HCF}_{2}-$ $\mathrm{SO}_{2} \mathrm{Na}$ with $\mathrm{Ph}_{2} \mathrm{PCl}$

硫基金属盐 $\mathrm{MSCF}_{2} \mathrm{H}$ 由于稳定性差的原因，难以直接合 成，至今没有文献报道. 2015 年，沈其龙课题组 ${ }^{[46]}$ 基于 $\mathrm{AgSCF}_{2} \mathrm{H}$ 不稳定的性质, 采取了用配体稳定银盐的策 略, 合成出了含有配体的二氟甲硫基银盐(SIPr)Ag$\left(\mathrm{SCF}_{2} \mathrm{H}\right)$, 该试剂也是第一个直接的亲核二氟甲硫基化 试剂. 作者将此试剂与芳香族重氮盐在非卤代铜盐的条 件下进行 Sandmeyer 反应，得到了一系列含二氟甲硫基 的芳香化合物. 该反应条件温和，应用广泛(Eq. 4).

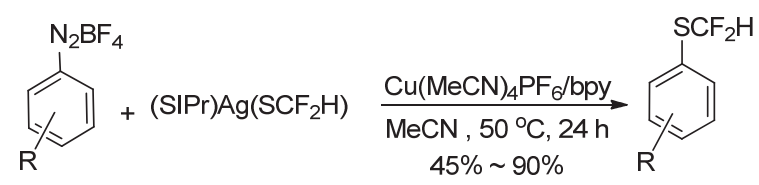

由于氟硣酸重氮盐价格昂贵，种类有限。作者随后 尝试了由芳胺制备重氮盐，不分离重氮盐中间体，一锅 法直接进行二氟甲硫基化反应，也获得了成功，极大地 提升了反应的应用价值(Eq. 5). 


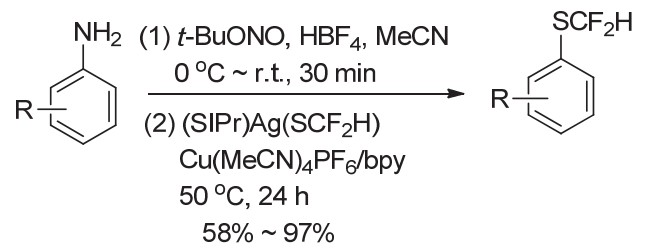

然而, 无论是四氟化硼重氮盐还是芳胺原位生成的 重氮盐, 在规模生产时都有潜在的爆炸危险. 在进行反 应时都需要小心操作, 这在很大程度上限制了该试剂的 应用范围. 鉴于此, 沈其龙课题组 ${ }^{[47]}$ 随后对该试剂的反 应底物和反应条件进行了进一步拓展, 成功实现了金属 钯催化 $(\mathrm{SIPr}) \mathrm{Ag}\left(\mathrm{SCF}_{2} \mathrm{H}\right)$ 与卤代烃的亲核二氟甲硫基化 反应. 众所周知, 钯催化的交叉偶联反应已经被广泛应 用于合成化学中，该类反应均涉及到还原消除，而含氟 砌块的还原消除通常情况下较难进行. 为了考察该反应 的进行过程, 作者首先用 xantphos 与 3-碘吡啶氧化加成 合成中间态, 再用 $(\mathrm{SIPr}) \mathrm{Ag}\left(\mathrm{SCF}_{2} \mathrm{H}\right)$ 与中间态进行配体 交换, 最后还原消除, 顺利地得到了目标产物(Scheme 13).

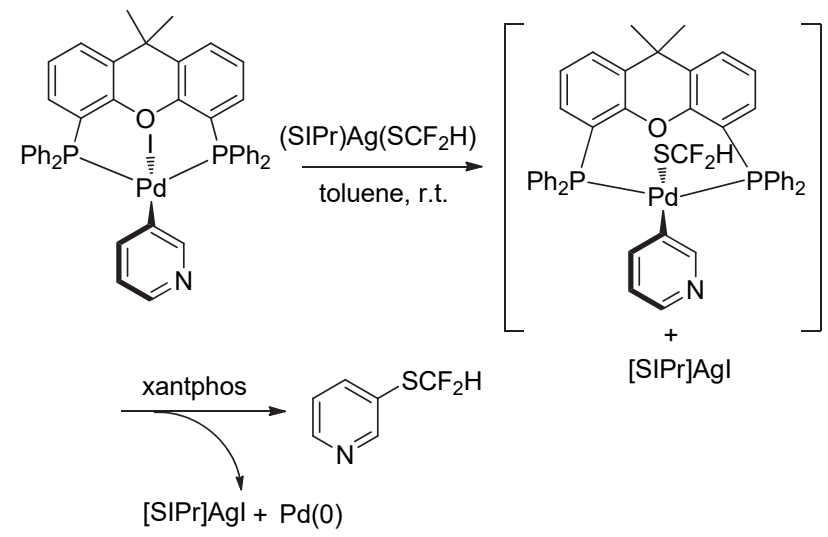

图式 $13 \mathrm{Pd}$ 催化的 $(\mathrm{SIPr}) \mathrm{Ag}\left(\mathrm{SCF}_{2} \mathrm{H}\right)$ 与杂环卤代烃的二氟甲 硫基化反应历程

Scheme 13 Palladium-catalyzed difluoromethylthiolation reaction mechanism of haloheteroaryl compounds with ( $\mathrm{SIPr}) \mathrm{Ag}\left(\mathrm{SCF}_{2} \mathrm{H}\right)$

随后沈其龙课题组 ${ }^{[47]}$ 从配体、溶剂几个方面优化了 反应条件. 发现 $(\mathrm{SIPr}) \mathrm{Ag}\left(\mathrm{SCF}_{2} \mathrm{H}\right)$ 在 $\mathrm{Pd}(\mathrm{dba})_{2}$ 与配体 xantphos 的共同作用下, 可以与吡啶、哒嗪、喹啉和嘧 啶等杂芳环溴代或碘代底物发生二氟甲硫基化反应, 收 率较高(Eq. 6).

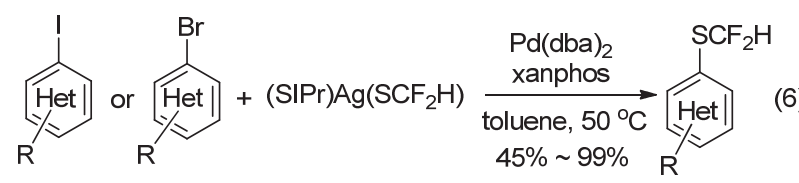

吡啶等缺电子杂环的溴代底物, 其反应活性远高于 苯环溴代底物, 更高于苯环氯代底物. 作者在利用以上
条件对卤代苯环进行类似反应研究时发现，该反应很难 进行. 在进行一系列深入研究后, 2018 年沈其龙课题 组 ${ }^{[48]}$ 报道了在 $\mathrm{Pd}(\mathrm{dba})_{2}$ 催化下, 以 BrettPhos 为配体, $(\mathrm{SIPr}) \mathrm{Ag}\left(\mathrm{SCF}_{2} \mathrm{H}\right)$ 可以与溴、氯以及 $\mathrm{OMs}$ 取代的苯环发 生取代反应，收率良好(Eq. 7).

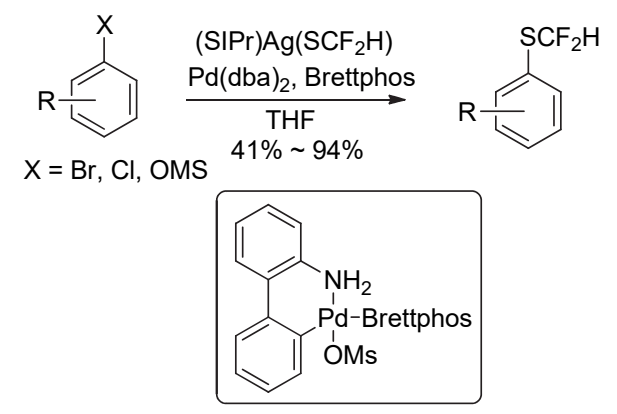

\section{3 自由基二氟甲硫基化}

自由基类型的直接二氟甲硫基化反应目前报道不 多，相比亲核和亲电反应，自由基反应的反应类型更加 丰富, 因此成为了一个研究热点与难点. 2016 年沈其龙 课题组 ${ }^{[49]}$ 利用二氟甲基苄硫醚、氯气与苯磺酸钠反应, 合成了第一个自由基类型的二氟甲硫基化试剂苯基磺 酰二氟甲基硫酯 $\left(\mathrm{PhSO}_{2} \mathrm{SCF}_{2} \mathrm{H}\right)$ (Eq. 8).

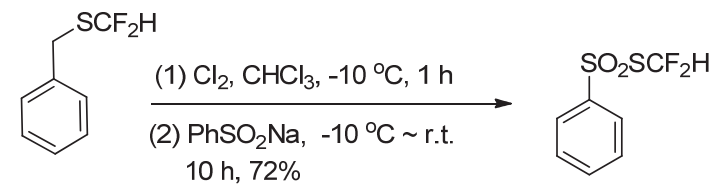

作者选用嗍酸底物对该试剂的应用条件进行了探 索，在硝酸银、十二烷基硫酸钠(SDS)与过硫酸钾的作用 下，有机嗍酸可以与上述试剂发生自由基反应，得到相 应的二氟甲硫基化合物. 反应条件对卤素、硝基、酰胺 和羰基等官能团均有很好的耐受性. 但由于二级和三级 脂肪族硼酸反应活性较低，且容易发生脱氢反应，极大 地限制了该试剂的应用范围. 作者随后研究了更加廉价 易得的反应底物羒酸类化合物. 由于在金属银盐作用下 羧酸很容易发生脱羧反应得到碳自由基，作者以此为出 发点, 研究了一系列羧基化合物的脱羧及其二氟甲硫基 自由基取代, 收率较好. 除取代反应外, 作者还研究了 $\mathrm{PhSO}_{2} \mathrm{SCF}_{2} \mathrm{H}$ 与烯烃的自由基加成，该反应以 $\mathrm{N}$-甲基吡 咯烷酮(NMP)或二甲基亚砜(DMSO)与水的混合物为溶 剂, 以 $\mathrm{AgNO}_{3}$ 和 $\mathrm{K}_{2} \mathrm{~S}_{2} \mathrm{O}_{8}$ 为引发剂, 室温下即可进行, 收 率最高可达 94\% (Scheme 14).

同年，作者对该试剂的应用进行了扩充. 在以上类 似的反应条件下, $\mathrm{PhSO}_{2} \mathrm{SCF}_{2} \mathrm{H}$ 可以与 1-取代环丁醇发 生开环加成反应，生成 $\gamma$-二氟甲硫基酮. 环丁醇的取代 基既可以是烷基也可以是芳环，收率较高. 随后，又将 


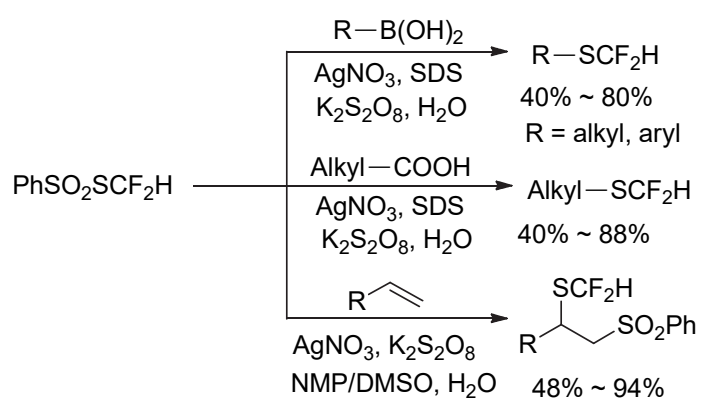

图式 $14 \mathrm{PhSO}_{2} \mathrm{SCF}_{2} \mathrm{H}$ 试剂的二氟甲硫基化反应

Scheme 14 Difluoromethylthiolation reaction using $\mathrm{PhSO}_{2} \mathrm{SCF}_{2} \mathrm{H}$

取代环丁醇引申到取代环丙醇、取代环戊醇和取代环庚 醇，均得到了相应的开环加成产物 ${ }^{[50]}$ (Eq. 9).

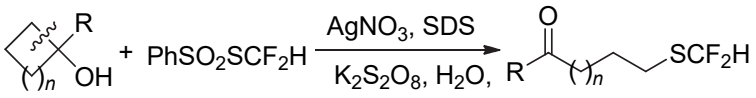

$$
\begin{aligned}
& 50^{\circ} \mathrm{C}, 6 \mathrm{~h} \\
& 40 \% \sim 90 \%
\end{aligned}
$$

2018 年 $\mathrm{Li}$ 课题组 ${ }^{[51]}$ 对 $\mathrm{PhSO}_{2} \mathrm{SCF}_{2} \mathrm{H}$ 参与的自由基 取代反应条件进行了拓展性研究. 发现在光照条件下, 用乙腈作溶剂, 加入催化量的四丁基碘化铵, 富电子的 芳环(包括吡咯、吲哚、多羟基苯环以及一些五元富电 子杂环)可以有效地发生自由基二氟甲硫基化反应，收 率较好(Eq. 10).

$$
\text { Ar }+\mathrm{PhSO}_{2} \mathrm{SCF}_{2} \mathrm{H} \underset{\substack{\mathrm{H} \mathrm{H}_{3} \mathrm{CN}, \mathrm{Ar} \text {, r.t. } \\ 40 \% \sim 93 \%}}{\stackrel{\mathrm{W} / \text { or w/o TBAI }}{\mathrm{CFL}(40 * 2 \mathrm{~W})}} \mathrm{Ar}^{\mathrm{SCF}_{2} \mathrm{H}}
$$

作者对反应机理进行了推测. 在光照条件下, $\mathrm{PhSO}_{2-}$ $\mathrm{SCF}_{2} \mathrm{H}$ 裂解成二氟甲硫基自由基与苯磺酰基自由基. 随 后二氟甲硫基自由基与芳环发生自由基取代反应，同时 苯磺酰基捕获氢自由基生成苯基亚磺酸(Scheme 15).

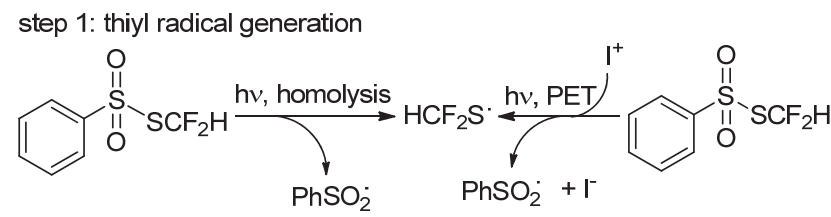

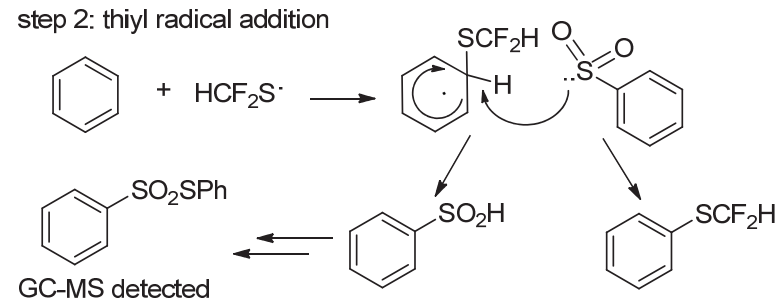

图式 $15 \mathrm{PhSO}_{2} \mathrm{SCF}_{2} \mathrm{H}$ 光照条件下的自由基二氟甲硫基化反 应机理推测

Scheme 15 Proposed mechanism for radical difluoromethylthiolation using $\mathrm{PhSO}_{2} \mathrm{SCF}_{2} \mathrm{H}$ under photocatalytic conditions
2018 年, 王永强课题组 ${ }^{[52]}$ 对 $\mathrm{PhSO}_{2} \mathrm{SCF}_{2} \mathrm{H}$ 的应用进 行了进一步拓展. 使用过氧叔丁醇( TBHP)作为引发剂, 将 $\mathrm{PhSO}_{2} \mathrm{SCF}_{2} \mathrm{H}$ 与醛反应，可以高收率地得到一系列羰 基二氟甲硫基化产物，反应条件温和，底物适用于各种 取代苯环，也适用于吡咯、吲哚、噻吩等芳香环以及部 分脂肪醛类化合物(Scheme 16).

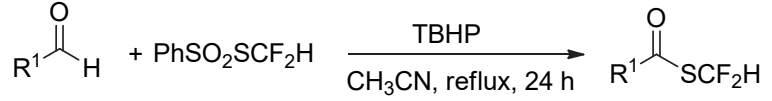

$$
\begin{aligned}
& 43 \% \text { } 88 \%
\end{aligned}
$$

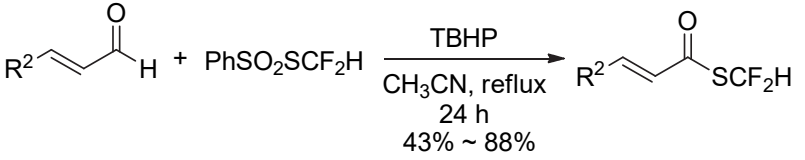

图式 $16 \mathrm{PhSO}_{2} \mathrm{SCF}_{2} \mathrm{H}$ 与醛的二氟甲硫基化反应 Scheme 16 Difluoromethylthiolation reaction of aldehyde with $\mathrm{PhSO}_{2} \mathrm{SCF}_{2} \mathrm{H}$

作者推测其反应机理可能是醛在叔丁基过氧化氢 (TBHP)引发下脱氢生成醛基自由基, 并与 $\mathrm{PhSO}_{2} \mathrm{SCF}_{2} \mathrm{H}$ 产生的二氟甲硫基自由基进行结合，得到产物。而 $\mathrm{PhSO}_{2} \mathrm{SCF}_{2} \mathrm{H}$ 的另一个片段苯磺酰基自由基，通过烯烃 捕获，从侧面证实了这一机理的合理性(Scheme 17).

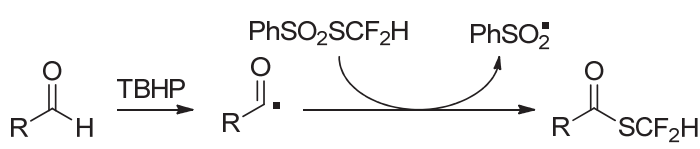

图式 $17 \mathrm{PhSO}_{2} \mathrm{SCF}_{2} \mathrm{H}$ 与酫的自由基二氟甲硫基反应机理推测 Scheme 17 Proposed mechanism for Radical difluoromethylthiolation using $\mathrm{PhSO}_{2} \mathrm{SCF}_{2} \mathrm{H}$ with aldehyde

2018 年, 徐政虎课题组 ${ }^{[53]}$ 系统地研究了 $\mathrm{PhSO}_{2} \mathrm{~S}$ $\mathrm{CF}_{2} \mathrm{H}$ 对炔烃的自由基加成反应. 在催化剂 $\mathrm{PPh}_{3} \mathrm{AuNTf}_{2}$ 和光照条件下，以 $\mathrm{Ru}(\mathrm{bpy})_{3} \mathrm{Cl}_{2}$ 为配体，炔烃可以与 $\mathrm{PhSO}_{2} \mathrm{SCF}_{2} \mathrm{H}$ 进行加成反应，得到二氟甲硫基与苯磺酰 基的双官能团烯烃. 在 $\mathrm{SmI}_{2}$ 作用下，苯磺酰基又会进一 步离去, 得到一系列新颖的含有二氟甲硫基烯的化合 物，收率中等(Scheme 18).

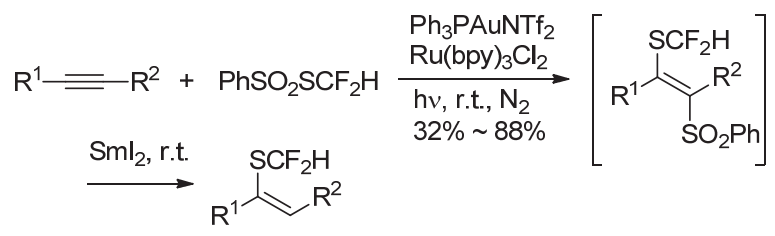

图式 $18 \mathrm{PhSO}_{2} \mathrm{SCF}_{2} \mathrm{H}$ 与炔的自由基加成反应

Scheme 18 Free radical addictive reaction of $\mathrm{PhSO}_{2} \mathrm{SCF}_{2} \mathrm{H}$ with alkyne

2003 年开始, 可见光促进的温和条件下产生碳自 由基的现象开始引起有机化学研究工作者的注意 ${ }^{[54]}$, 
可见光催化的脂肪族羧酸的自由基脱羧反应也有了进 展 $^{[55]} .2016$ 年, Glorius 课题组 ${ }^{[56]}$ 利用二氟甲硫基邻苯二 甲酰亚胺 $\left(\mathrm{PhthSCF}_{3}\right)$ 与脂肪族羧酸在可见光条件下脱 羧, 合成了一系列脂肪族二氟甲硫基化合物(Eq. 11).<smiles>[X][SH+]([S-])N1C(=O)c2ccccc2C1=O</smiles>

$$
\begin{aligned}
& \underset{\substack{\text { cat. } \mathrm{PCl}, \mathrm{CsOBz} \\
\mathrm{C}_{6} \mathrm{H}_{5} \mathrm{~F}, \text { bule LED } \\
14 \mathrm{~h}}}{\mathrm{R}-\mathrm{SCF}_{2} \mathrm{H}} \\
& 49 \% \sim 90 \%
\end{aligned}
$$

作者推测其机理可能是脂肪族羧酸在光引发剂和 可见光共同作用下, 先脱羧生成碳自由基, 随后碳自由 基与 $\mathrm{PhthSCF}_{3}$ 反应得到二氟甲硫基产物(Scheme 19).

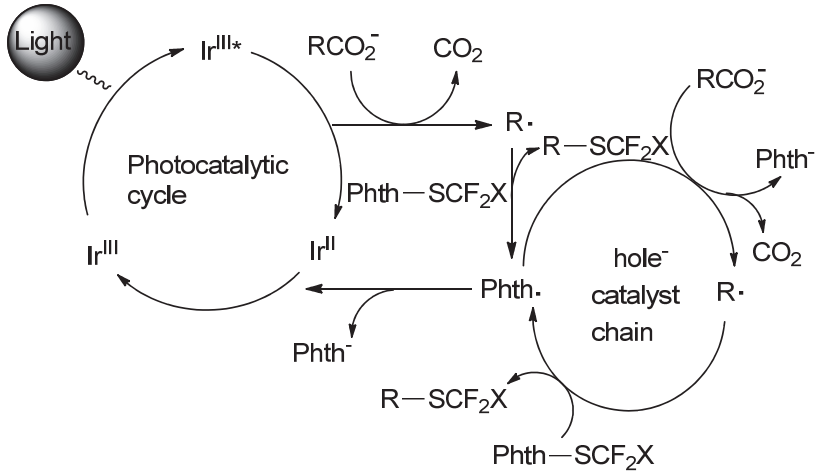

图式 19 二氟甲硫基邻苯二甲酰亚胺与羧酸在可见光下的自 由基脱羧二氟甲硫基化反应机理

Scheme 19 Proposed mechanism for visible light-promoted decarboxylative difluoromethylthiolation of alkyl carboxylic acids with $\mathrm{PhthSCF}_{3}$

2018 年, 朱成建课题组 ${ }^{[57]}$ 研究发现, 甲氧甲基 $(\mathrm{MOM})$ 保护的叔醇可以被 $\mathrm{PhthSCF}_{3}$ 取代, 生成三氟甲 硫基化合物, 推测该过程为自由基反应历程. 在此基础 上. 作者对该类反应向二氟甲硫基化方向进行了衍生. 在光照条件下, 用 MOM 保护的叔醇与 $\mathrm{PhthSCF}_{2} \mathrm{H}$ 反应, 也以中等收率得到了目标产物(Eq. 12).

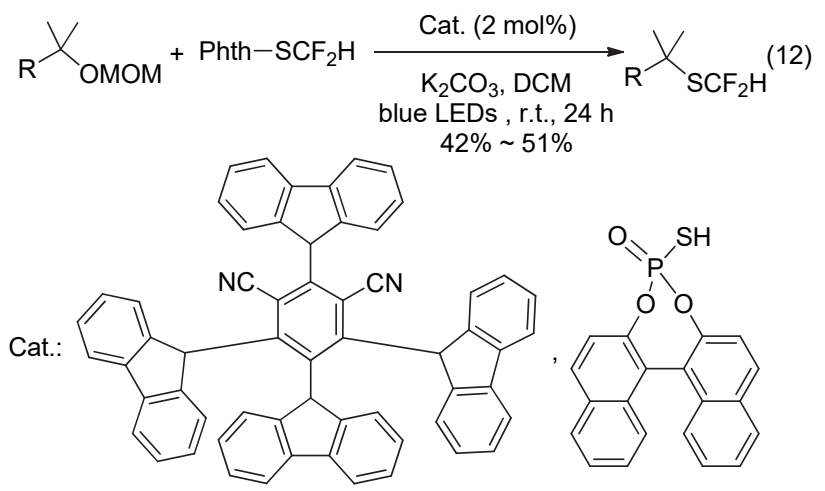

\section{4 不含硫底物的其他二氟甲硫化策略}

2015 年, Goossen 课题组 ${ }^{[2]}$ 报道了一种通过亲核取 代一锅法引入二氟甲硫基的方法. 其采用的策略是, 先
向分子中引入 $\mathrm{SCN}$ 基团; 同时利用廉价易得的 $\mathrm{TMSCF}_{2} \mathrm{H}$ 作为氟源，将其与 $\mathrm{CuSCN}$ 进行反应，原位生 成 $\mathrm{CuCF}_{2} \mathrm{H}$; 最后用 $\mathrm{CuCF}_{2} \mathrm{H}$ 将 $\mathrm{SCN}$ 上的 $\mathrm{CN}$ 取代, 生 成 $\mathrm{SCF}_{2} \mathrm{H}$ 产物. 虽然整个反应分为两个部分, 但通过优 化反应条件，成功地实现了一锅法直接合成一系列 $\mathrm{SCF}_{2} \mathrm{H}$ 类化合物. 文献报道的底物分为两大类: 第一类 是脂肪族的溴化物和 $\mathrm{OMs}$ 取代物; 第二类是芳香族的 重氮盐. 值得一提的是, 无论芳环上有吸电子的取代基 还是供电子的取代基，只要 $\mathrm{SCN}$ 能够成功地连接到芳 环上, $\mathrm{CN}$ 基团均可以被 $\mathrm{CF}_{2} \mathrm{H}$ 基团取代(Scheme 20).

$$
\begin{array}{cl}
\mathrm{R}-\mathrm{Br} / \mathrm{OMs} \quad \stackrel{\begin{array}{l}
\text { (1) } \mathrm{NaSCN} \\
\text { (2) CuSCN, CsF, } \mathrm{TMSCF}_{2} \mathrm{H}
\end{array}}{\mathrm{DMF}, 0^{\circ} \mathrm{C} \sim \text { r.t. }} \\
61 \% \sim 99 \%
\end{array}
$$

图式 $20 \mathrm{CuSCN}$ 与 $\mathrm{TMSCF}_{2} \mathrm{H}$ 一锅法合成二氟甲硫基化合物 Scheme 20 One pot synthesis of $\mathrm{SCF}_{2} \mathrm{H}$-containing molecules by using $\mathrm{CuSCN}$ and $\mathrm{TMSCF}_{2} \mathrm{H}$

2015 年, Goossen 小组 ${ }^{[58]}$ 将这一合成策略所适用的 底物进行了进一步拓展, 并对合成工艺进行了优化与改 进. 选用了新的廉价易得的 $\mathrm{SCN}$ 化试剂 $N$-硫氰基丁二 酰亚胺(NTS), 对富电子的芳环氢进行亲电取代, 接着 再用 $\mathrm{TMSCF}_{2} \mathrm{H}$ 将氰基取代，收率中等(Eq. 13).

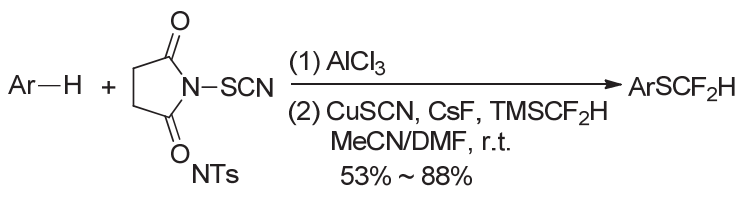

硫脲是一种经济稳定的含硫有机物，近些年，有许 多文献报道了用硫脲作为硫源构建含硫有机物的方法. 我们课题组 ${ }^{[59]}$ 在这些工作的基础上, 发展了一种一锅 法制备二氟甲硫基化合物的有效方法. 该法以硫脲作为 硫源，用水与二氧六环的混合液作溶剂，在碘/碘化钾的 作用下，取代吲哚与取代吡咯先生成含有颈基的中间产 物, 该中间体不需分离, 直接与溴二氟甲基亚磷酸二乙 酯 $\left[\mathrm{BrF}_{2} \mathrm{CPO}(\mathrm{OEt})_{2}\right]$ 进行二氟甲基化，得到二氟甲硫基 产物, 收率最高可以达到 94\% (Scheme 21).

2017 年, 肖吉昌课题组 ${ }^{[60]}$ 研究发现, $\mathrm{Ph}_{3} \mathrm{P}^{+} \mathrm{CF}_{2} \mathrm{CO}_{2}{ }^{-}$ (PFDA) 与硫 $\mathrm{S}_{8}$ 在 $80{ }^{\circ} \mathrm{C}$ 下反应 $30 \mathrm{~min}$ 得到二氟硫酮. 当 反应体系中同时存在相邻的杂原子时，可以立即与二氟 硫酮反应得到硫嫝类化合物，同时研究人员还捕捉到了 少量含有 $\mathrm{SCF}_{2} \mathrm{H}$ 的副产物. 通过对反应溶剂、PFDA 用 量、反应温度及碱等反应条件的篮选和优化后，最终以 
<smiles>[R]c1ccc2[nH]ccc2c1</smiles><smiles>NC(=S)OCCOCCO</smiles><smiles>[R]c1ccc2[nH]cc(SC(=[NH+])N)c2c1</smiles>

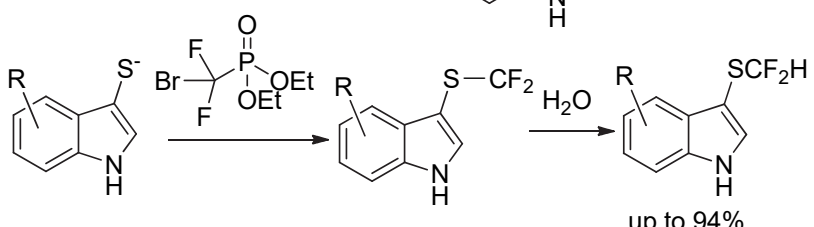

up to $94 \%$

图式 21 硫艮作为硫源引入二氟甲硫基

Scheme 21 Difluoromethylthiolation using thiourea as S source 中等收率得到了一系列取代硫嫝结构的二氟甲硫基产 物(Eq. 14).

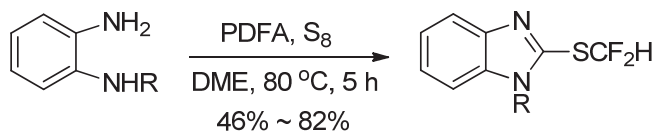

作者推测了可能的反应历程: 首先 PFDA 分解得到 二氟卡宾, 并与 $\mathrm{S}_{8}$ 结合得到二氟硫酮; 随后二氟硫酮与 体系内的 $\mathrm{N}$ 原子结合, 生成取代硫脲结构的中间体; 最 后该中间体与多余的二氟卡宾结合，得到二氟甲硫基产 物(Scheme 22).

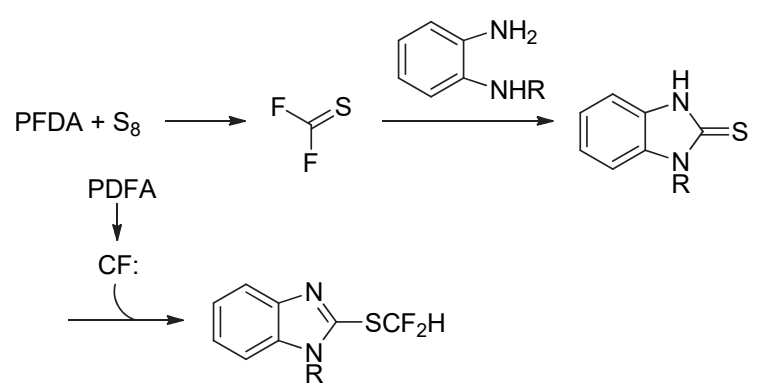

图式 22 取代邻苯二胺在 PFDA 和 $\mathrm{S}_{8}$ 条件下的二氟甲硫基化 反应历程

Scheme 22 Proposed mechanism for difluoromethylthiolation of vicinal diamine with PFDA and $\mathrm{S}_{8}$

\section{5 含有 $S_{C F} G$ 切块的化合物的直接合成}

含有 $\mathrm{SCF}_{2} \mathrm{G}$ 砌块的化合物也是二氟甲硫基化合物 家族的一个重要组成部分, 由于其同时含有 $\mathrm{SCF}_{2}$ 与 $\mathrm{G}$ 两个官能团, 其潜在的应用价值不可忽视. 同时两个官 能团的存在也使得该类分子合成难度较 $\mathrm{SCF}_{2} \mathrm{H}$ 大. 近 年来, 随着直接二氟甲硫基化反应的深入研究, 直接合 成含有 $\mathrm{SCF}_{2} \mathrm{G}$ 的方法也陆续被报道.

2018 年, Gooßen 课题组 ${ }^{[61]}$ 对取代二氟甲硫基进行 了拓展性研究, 在 $\mathrm{CsCO}_{3}$ 与 $\mathrm{NaSCN}$ 存在下, 芳香族重 氮化合物可以一锅法与 $\mathrm{CuSCN}$ 及 $\mathrm{TMSCF}_{2} \mathrm{PO}(\mathrm{OEt})_{2}$ 反 应, 得到一系列含有二氟甲硫基羧亚磷酸乙酯 $\left[\mathrm{SCF}_{2} \mathrm{PO}-\right.$ $\left.(\mathrm{OEt})_{2}\right]$ 的底物，收率中等(Eq. 15).

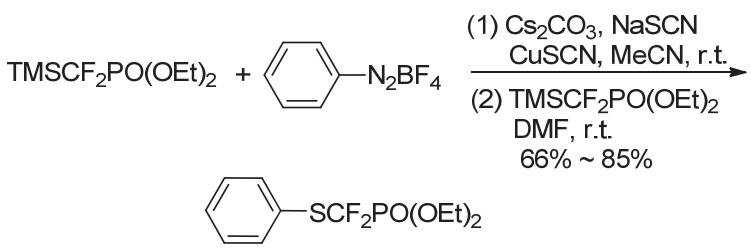

(15)

赵刚课题组 ${ }^{[62]}$ 在这方面也做了一些工作，他们采 用一锅法, 将 $\mathrm{TMSCF}_{2} \mathrm{COOMe} 、 \mathrm{CuSCN}$ 与芳香族重氮 盐或脂肪族溴代底物进行反应，得到了一系列含有二氟 甲硫基羧酸乙酯 $\left(\mathrm{SCF}_{2} \mathrm{CO}_{2} \mathrm{Et}\right)$ 的产物，反应条件温和， 收率较高(Scheme 23).

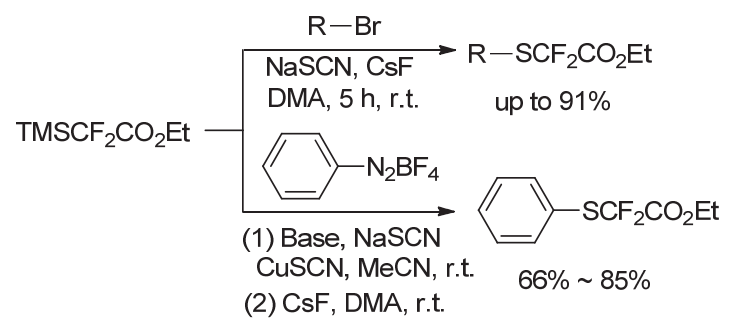

图式 $23 \mathrm{TMSCF}_{2} \mathrm{COOEt}$ 试剂合成含 $\mathrm{SCF}_{2} \mathrm{COOEt}$ 官能团分子 的反应

Scheme 23 Synthesis of $\mathrm{SCF}_{2} \mathrm{COOEt}$-containing molecules by using $\mathrm{TMSCF}_{2} \mathrm{COOEt}$

2017 年, 沈其龙课题组 ${ }^{[63]}$ 进一步开发了另一个含 有二氟甲硫基羧酸酯的亲电试剂邻苯二甲酰胺二氟甲 硫基乙酸酯(PhthNSCF 2 COOEt), 并深入研究了适合该 试剂的反应条件. 研究发现, 常见的合成 $\mathrm{CF}_{3} \mathrm{~S}$ 或 $\mathrm{HCF}_{2} \mathrm{~S}$ 官能团的亲电取代的反应条件，如 $\mathrm{TMSCl}$ 或 $\mathrm{BF}_{3} \cdot \mathrm{Et}_{2} \mathrm{O}$ 都不能很好地将二氟甲硫基羧酸酯官能团引入目标分 子. 而利用溴化镁作为催化剂, 则可以在亲电芳环和硫 醇上进行反应，收率适中。 与 $\mathrm{PhthNSCF}_{2} \mathrm{H}$ 类似，该试 剂也可以与 $\beta$ 二羰基化合物在弱碱 $\left(\mathrm{K}_{2} \mathrm{CO}_{3}\right)$ 条件下进行 反应(Scheme 24).

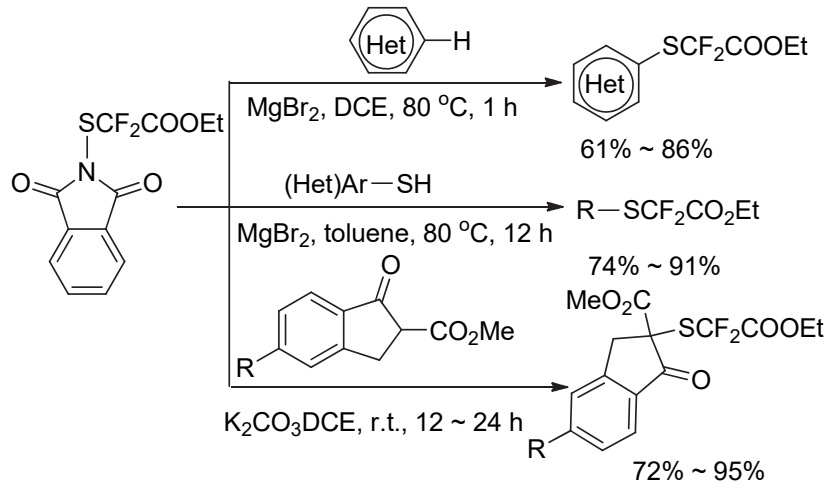

图式 24 PhthNSCF $F_{2}$ COOEt 试剂合成含 $\mathrm{SCF}_{2} \mathrm{COOEt}$ 官能团 分子的反应

Scheme 24 Synthesis of $\mathrm{SCF}_{2}$ COOEt-containing molecules by using $\mathrm{PhthNSCF}_{2} \mathrm{COOEt}$ 
$\mathrm{ArNHSCF}_{2} \mathrm{R}$ 类型的直接二氟甲硫基化试剂也是最 近两年的一个研究热点. 由于 $\mathrm{N}-\mathrm{S}$ 键不稳定, 在酸性 条件下断裂后, 可以将官能团 $\mathrm{SCF}_{2} \mathrm{R}$ 直接引入富电子底 物中。2016 年, Billard 课题组 ${ }^{[64]}$ 首先开发了 $\mathrm{PhNHSCF}_{2} \mathrm{SO}_{2} \mathrm{Ph}$. 由于原路线收率不高, 一年后该课题 组对其合成路线进行了改进 ${ }^{[65]}$, 使得此试剂的合成更 加简单高效(Scheme 25).

Billard 最初研究了该试剂与双键的亲电加成反应, 在对甲苯磺酸或三氟化硼乙醚催化作用下, 不饱和键可 以与该试剂生成双官能化产物, 但两个条件收率都不 高, 推测可能是因为官能团 $\mathrm{SCF}_{2} \mathrm{PhSO}_{2}$ 空间位阻较大. 随后 Billard 又尝试了富电子芳环吲哚、吡咯以及羰基 $\alpha$ 氢的 ${ }^{[66]}$ 的亲电取代反应研究, 收率较高. 此外, 作者对
$\mathrm{PhSO}_{2}$ 的离去反应条件也做了深入研究, 在催化量的 $\mathrm{I}_{2}$ 或 5 equiv.的 TMSCl 存在下, $\mathrm{PhSO}_{2}$ 可以高收率地发生离 去反应, 得到 $\mathrm{SCF}_{2} \mathrm{H}$ 化合物(Scheme 26).

2016 年, Besset 课题组 ${ }^{[67]}$ 通过硫氰化均三苯胺、 $\mathrm{CuSCN}$ 与 $\mathrm{TMSCF}_{2} \mathrm{PO}(\mathrm{OEt})_{2}$ 反应, 合成了另一个 $\mathrm{ArNHSCF}_{2} \mathrm{R}$ 类型的二氟甲硫基化试剂, 其中 $\mathrm{R}=$ $\mathrm{PO}(\mathrm{OEt})_{2}, \mathrm{Ar}$ 为均三甲苯. 该试剂在路易斯酸的活化下, 与一系列的富电子底物发生反应, 包括吲哚、吡咯、富 电子苯环、羰基和硫酚等, 得到相应的二氟甲硫醚产物, 在合成上具有较大的应用价值. 此外, 由于试剂中的 $\mathrm{Ar}$ 为三甲基苯, 本身有较大的空间位阻，使得该试剂较其 他小位阻的苯胺有更高的活性，从而可以合成其它 $\mathrm{N}$ 取 代的 $\mathrm{SCF}_{2} \mathrm{PO}(\mathrm{OEt})_{2}$ 化合物(Scheme 27).

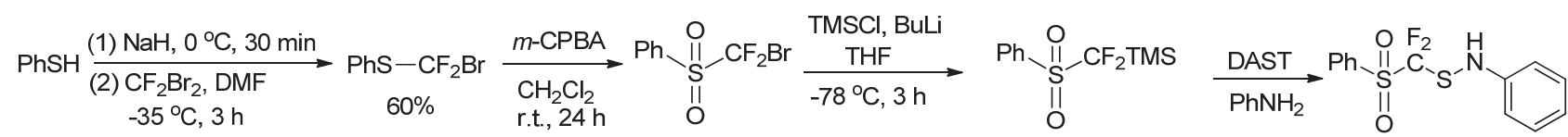

$$
\begin{aligned}
& \mathrm{PhSH} \underset{\text { (2) } \mathrm{CF}_{3} \mathrm{SiMe}_{3}(2.5 \text { equiv.) }}{\stackrel{(1)}{\longrightarrow}} \\
& \mathrm{RuCl}_{3}, \mathrm{NaIO}_{4} \mid \begin{array}{c}
\mathrm{CH}_{3} \mathrm{CN} / \mathrm{CCl}_{4} / \mathrm{H}_{2} \mathrm{O} \\
0^{\circ} \mathrm{C} \sim \text { r.t., } 2.5 \mathrm{~h} \\
75 \%
\end{array} \\
& \text { DMF, } 25^{\circ} \mathrm{C} \\
& \mathrm{PhS}-\mathrm{CF}_{2} \mathrm{TMS} \\
& 99 \%
\end{aligned}
$$

图式 $25 \mathrm{PhNHSCF}_{2} \mathrm{SO}_{2} \mathrm{Ph}$ 的制备过程

Scheme 25 Preparation of $\mathrm{PhNHSCF}_{2} \mathrm{SO}_{2} \mathrm{Ph}$

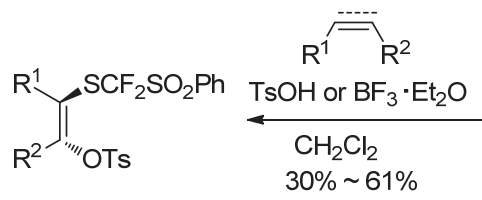<smiles>O=S(=O)(CSNc1ccccc1)c1ccccc1</smiles>

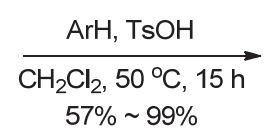

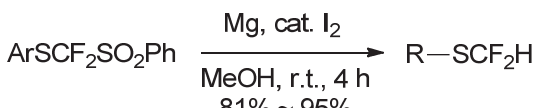

图式 $26 \mathrm{PhNHSCF}_{2} \mathrm{SO}_{2} \mathrm{Ph}$ 试剂合成含 $\mathrm{SCF}_{2} \mathrm{SO}_{2} \mathrm{Ph}$ 官能团分子的反应 Scheme 26 Synthesis of $\mathrm{SCF}_{2} \mathrm{SO}_{2} \mathrm{Ph}$-containing molecules by using $\mathrm{PhNHSCF}_{2} \mathrm{SO}_{2} \mathrm{Ph}$
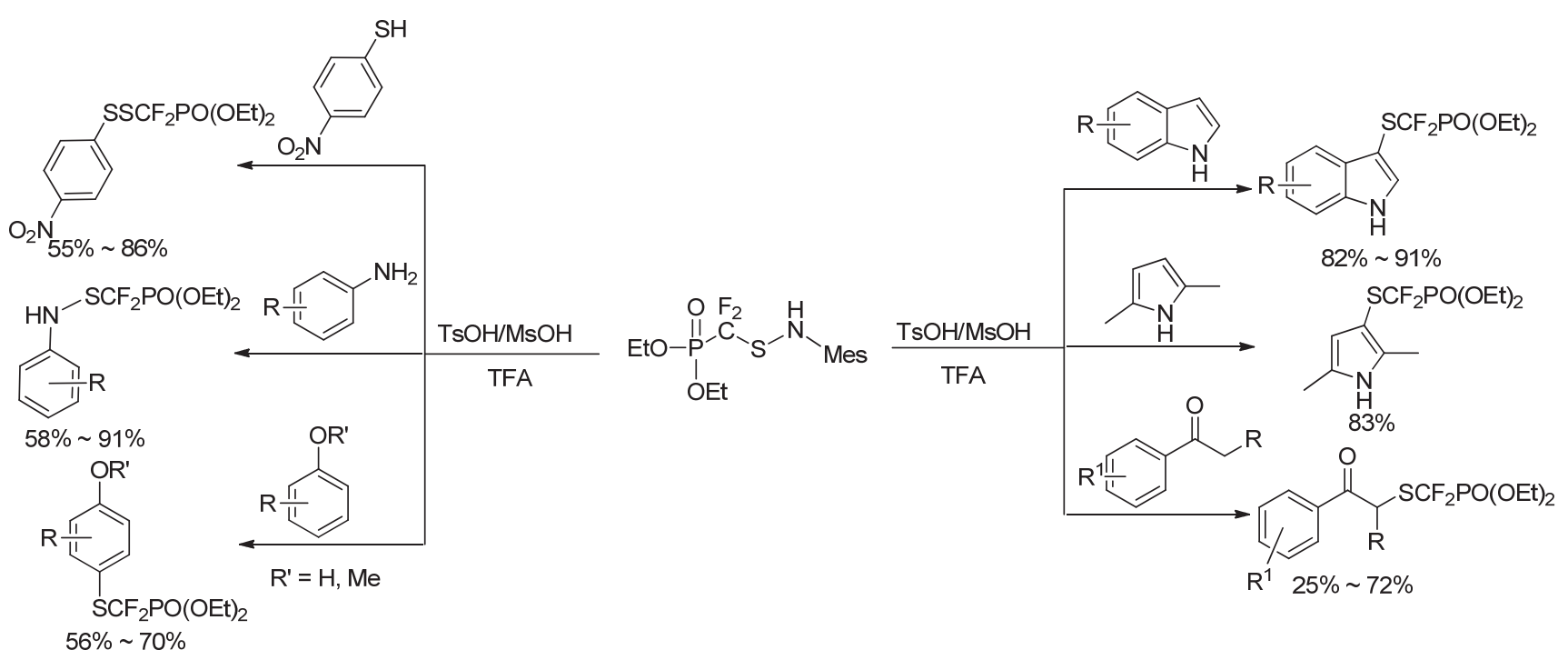

图式 $27 \mathrm{MesNHSCF}_{2} \mathrm{P}(\mathrm{O})(\mathrm{OEt})_{2}$ 试剂合成含 $\mathrm{SCF}_{2} \mathrm{P}(\mathrm{O})(\mathrm{OEt})_{2}$ 官能团分子的反应 Scheme 27 Synthesis of $\mathrm{SCF}_{2} \mathrm{P}(\mathrm{O})(\mathrm{OEt})_{2}$-containing molecules by using MesNHSCF $2 \mathrm{P}(\mathrm{O})(\mathrm{OEt})_{2}$ 


\section{2 一氟甲硫基化反应}

与二氟甲硫基化反应的发展情况类似，单氟甲硫基 化反应主要分为两大类. 传统的单氟甲硫基化方法包括 甲硫醚 ${ }^{[68 ~ 70]}$ 与取代甲硫醚(主要包括卤代 ${ }^{[71,72]}$ 、磺酸

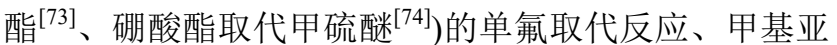
砜的还原氟化 ${ }^{[75 ~ 77]}$ 以及含有放基化合物的单氟烷基化 反应 ${ }^{[78 ~ 81]}$. 与二氟甲硫基化反应情况类似, 以上这些方 法虽然具有反应条件简单等特点, 但是含硫底物本身数 量有限, 有些底物制备困难, 极大地限制了其拓展能力. 鉴于此, 从 2017 年开始, 关于直接的一氟甲硫基化反应 的研究报道开始陆续出现(Scheme 28).

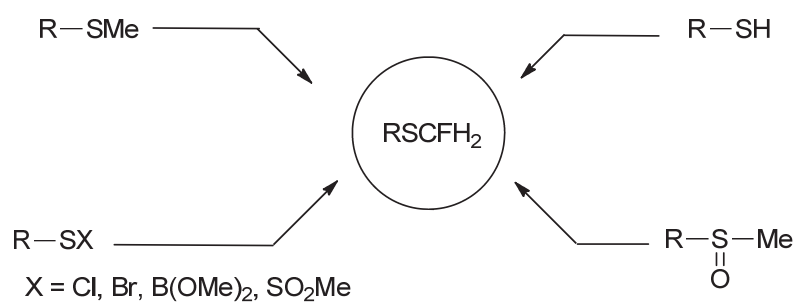

图式 28 间接引入一氟甲硫基的方法

Scheme 28 Indirect mehods of monofluoromethylthiolation

\section{1 亲电一氟甲硫基化反应}

1996 年, O'Mahony 团队 ${ }^{[82]}$ 在研究三唑类杀虫活性 分子时, 利用单氟甲基硫醇与不含硫的三唑底物发生取 代反应，直接得到了一个含有单氟甲硫基基团的活性分 子. 文章没有详细地叙述实验细节, 但单氟甲硫醇本身 制备条件苛刻 ${ }^{[83]}$, 需要氟气, 且收率不高, 很难进行推 广(Eq. 16).
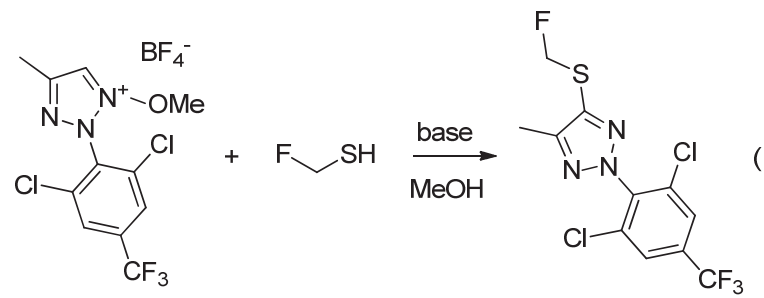

\section{2 自由基一氟甲硫基化反应}

2017 年, 沈其龙小组 ${ }^{[84}$ 利用硫代苯磺酸钠与一氟 卤代烷以 $N, N-$ 二甲基甲酰胺(DMF)作溶剂, 高收率地合 成出了一个单氟甲硫基化试剂单氟甲硫基苯磺酸酯 $\left(\mathrm{PhSO}_{2} \mathrm{SCFH}_{2}\right)$, 并对其反应条件进行了研究. 研究表明 在硫酸铜催化下，以碳酸氢钠作碱，该试剂在室温条件 下即可与芳基硼酸发生取代反应，收率最高可达 93\%, 官能团适用性较广, 醛基、硝基、卤代烃、酯基和氰基 等均不受影响. 同时, 作者也对单氟甲硫基苯磺酸酯对 烯烃的加成反应进行了报道. 在硝酸银的催化下，以过
硫酸钾为引发剂, $\mathrm{NMP} /$ 水为溶剂, 该试剂在室温下即可 与烯烃进行加成反应，生成苯磺酰基与单氟甲硫基的双 官能化产物，收率较高(Scheme 29).

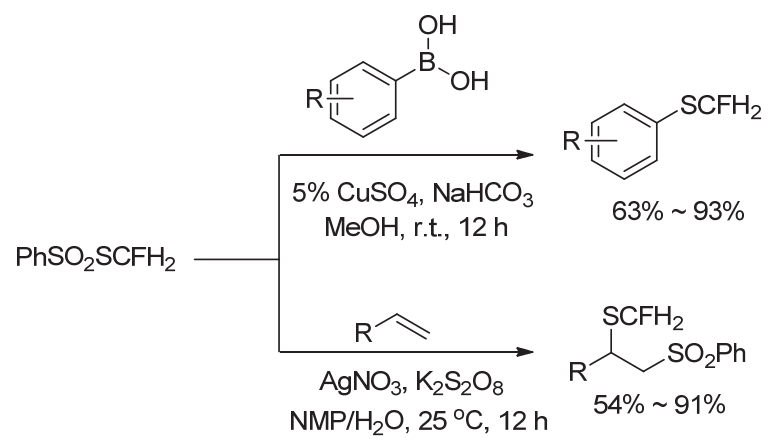

图式 $29 \mathrm{PhSO}_{2} \mathrm{SCFH}_{2}$ 试剂的一氟甲硫基化反应 Scheme 29 Monofluoromethylthiolation reaction using $\mathrm{PhSO}_{2}-$ $\mathrm{SCFH}_{2}$

作者对反应类型进行了推测研究, 在反应中加入自 由基抑制剂四甲基哌啶氮氧化物(TEMPO), 会彻底抑制 反应的进行，加入 2,6-二叔丁基-4-甲基苯酚与二硝基苯 也可以部分程度地抑制反应. 同时, 利用经典的自由基 Clock 反应条件，以苯基环丙级乙烯为底物，可以得到 开环重排加成双官能化产物，也进一步证实了自由基反 应历程的可能性(Eq. 17).

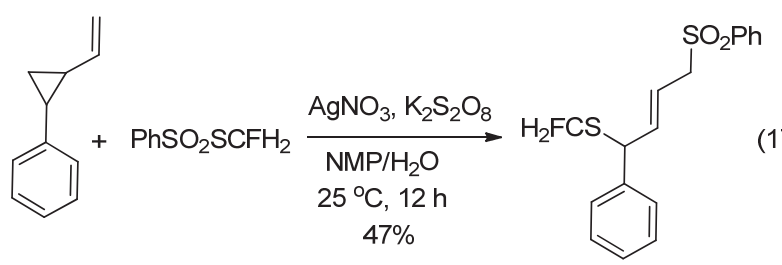

2018 年王永强课题组 ${ }^{[85]}$ 对单氟甲硫基苯磺酸酯的 应用进行了扩充. 以偶氮二异戊腈 $(A M B N)$ 为引发剂, 二氯乙烷为溶剂，在回流下用醛与单氟甲硫基苯磺酸酯 进行反应，得到一系列单氟甲硫基酯，该反应对芳香醛 与脂肪醛均有效，收率较好，最高可达 $91 \%$. 同时，作 者发现使用 TEMPO 会抑制反应，而通过光照引发的方 式即使不加热也可以得到产物，证实该反应是自由基历 程(Eq. 18).

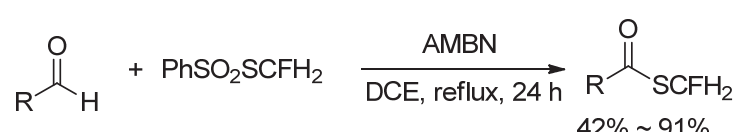

2018 年, 我们课题组 ${ }^{[86]}$ 发展了一种新型的一氟甲 硫基 Bunte 盐 $\mathrm{CFH}_{2} \mathrm{SSO}_{3} \mathrm{Na}$. 该试剂利用一氟碘甲烷与 硫代硫酸钠在甲醇水体系中制得. 与前述试剂相比，该 试剂原子经济性更高. 在硫酸铜催化下, 苯胺制得的重 氮盐无需分离即可与 $\mathrm{CFH}_{2} \mathrm{SSO}_{3} \mathrm{Na}$ 发生反应，以中等收 率制得单氟甲硫基取代的芳香化合物，同时该试剂也可 
以与苯硫酚发生取代反应, 得到一系列含有 $\mathrm{SSCH}_{2} \mathrm{~F}$ 官 能团的化合物(Scheme 30).

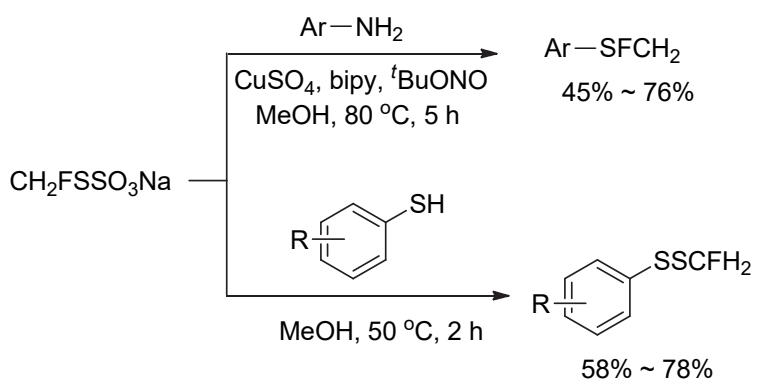

图式 $30 \mathrm{CH}_{2} \mathrm{FSSO}_{3} \mathrm{Na}$ 参与的自由基单氟甲硫基化反应 Scheme 30 Radical monofluoromethylthiolation using $\mathrm{CH}_{2} \mathrm{FSSO}_{3} \mathrm{Na}$

\section{3 结论与展望}

综述了近年来直接二氟甲硫基化反应与直接一氟 甲硫基化反应的研究情况, 对各种新试剂的制备方法进 行了概述, 对反应类型进行了归类, 并对反应机理进行 了总结. 虽然直接二氟甲硫基化与直接一氟甲硫基化反 应的研究已经有了的进步，但仍然面临试剂制备困难、 价格昂贵、原子经济性差及适用范围不足等缺点. 因此 发展绿色、高效且经济的二氟甲硫基化与一氟甲硫基化 的试剂和方法, 仍然化学工作者面临的巨大挑战.

\section{References}

[1] Erickson, J. A.; McLoughlin, J. I. J. Org. Chem. 1995, 60, 1626

[2] Bayarmagnai, B.; Matheis, C.; Jouvin, K.; Goossen, L. J. Angew. Chem., Int. Ed. 2015, 54, 5753

[3] Fourie, J. J.; Horak, I. G.; Redondo, P. V. Vet. Rec. 2010, 167, 442

[4] Morita, K.; Ide, K.; Hayase, Y.; Takahashi, T.; Hayashi, Y. Agric. Biol. Chem. 1987, 51, 1339.

[5] Yagupolskii, L. M.; Maletina, I. I.; Petko, K. I.; Fedyuk, D. V.; Handrock, R.; Shavaran, S. S.; Klebanov, B. M.; Herzig, S. J. Fluorine Chem. 2001, 109, 87.

[6] Shimizu, K. Jpn. J. Antibiot. 1988, 12, 1809

[7] Kettner, M.; Krčmery, V.; Bonini, P.; Cichero, P.; Ossi, C.; Grazioli, V. Infection 1991, 19, 253

[8] Burkholder, C. R.; Dolbier, W. R.; Medebielle, M. J. Fluorine Chem. 2000, 102, 369

[9] Boiko, V. N. Beilstein J. Org. Chem. 2010, 6, 880

[10] Ashton, M. J.; Lawrence, C.; Karlsson, J. A.; Stuttle, K. A. J.; Newton, C. G.; Vacher, B. Y. J.; Webber, S.; Withnall, M. J. J. Med. Chem. 1996, 39, 4888.

[11] Howland, W. C. Clin. Exp. Allergy 1996, 26, 18.

[12] Jung, M.; Wahl, A. F.; Neupert, W.; Geisslinger, G.; Senter, P. D. Pharm. Pharmacol. Commun. 2000, 6, 217.

[13] Hine, J.; Porter, J. J. J. Am. Chem. Soc. 1957, 79, 5493.

[14] Prakash, G. K. S.; Ni, C. F.; Wang, F.; Hu, J. B.; Olah, G. A. Angew. Chem., Int. Ed. 2011, 50, 2559.

[15] Zhao, Y. C.; Huang, W. Z.; Zhu, L. G.; Hu, J. B. Org. Lett. 2010, 12 , 1444.

[16] Kudo, N.; Yondea, A.; Sato, K.; Honma, T.; Sugai, S. Chem. Pharm. Bull. 2000, 48, 509.

[17] Wang, W. Q.; Yu, Q. W.; Zhang, Q.; Li, J. W.; Hui, F.; Yang, J. M.; Lü, J. Chin. J. Org. Chem. 2018, 38, 1569 (in Chinese).
(王为强, 余秦伟, 张前, 李江伟, 惠丰, 杨建明, 吕剑, 有机化 学, 2018, 38, 1569.)

[18] Fuchibe, K.; Bando, M.; Takayama, R.; Ichikawa, J. J. Fluorine Chem. 2015, 171, 133.

[19] Zafrani, Y.; Sod-Moriah, G.; Segall, Y. Tetrahedron 2009, 65, 5278.

[20] Zhang, W.; Wang, F.; Hu, J. B. Org. Lett. 2009, 11, 2109.

[21] Wang, F.; Huang, W. Z.; Hu, J. B. Chin. J. Chem. 2011, 29, 2717.

[22] Prakash, G. K. S.; Zhang, Z.; Wang, F.; Ni, C. F.; Olah, G. A. J. Fluorine Chem. 2011, 132, 792.

[23] Fier, P. S.; Hartwig, J. F. Angew. Chem., Int. Ed. 2013, 52, 2092.

[24] Li, L. C.; Wang, F.; Ni, C. F.; Hu, J. B. Angew. Chem., Int. Ed. 2013, 52, 12390 .

[25] Han, J. B.; Qin, H. L.; Ye, S. H.; Zhu, L; Zhang, C. P. J. Org. Chem. 2016, 81, 2506.

[26] Howard, J. L.; Schotten, C.; Alston, S. T.; Browne, D. L. Chem. Commun. 2016, 52, 8448.

[27] Thomoson, C. S.; Dolbier, Jr. W. R. J. Org. Chem. 2013, 78, 8904.

[28] Mehta, V. P.; Greaney, M. F. Org. Lett. 2013, 15, 5036.

[29] Deng, X. Y.; Lin, J. H.; Zheng, J.; Xiao, J. C. Chem. Commun. 2015, 51, 8805.

[30] Fujiwara, Y.; Dixon, J. A.; Rodrigue, R. A.; Baxter, R. D.; Dixon, D. D.; Collins, M. R.; Blackmond, D. G.; Baran, P. S. J. Am. Chem. Soc. 2012, 134, 1494.

[31] Lin, Y. M.; Yi, W. B.; Shen, W. Z; Lu, G. P. Org. Lett. 2016, 18, 592.

[32] Zhu, D. H.; Gu, Y.; Lu, L.; Shen, Q. L. J. Am. Chem. Soc. 2015, 137, 10547.

[33] Zhu, D. H.; Hong, X.; Li, D. Z.; Lu, L.; Shen, Q. L. Org. Process Res. Dev. 2017, 21, 1383.

[34] Yang, Y. D.; Azuma, A.; Tokunaga, E.; Yamasaki, M.; Shiro, M.; Shibata, N. J. Am. Chem. Soc. 2013, 135, 8782.

[35] Arimori, S.; Matsubara, O.; Takada, M.; Shibata, N. R. Soc. Open Sci. 2016, 3, 1601021

[36] Gondo, S.; Matsubara, O.; Chachignon, H.; Sumii, Y.; Cahard, D.; Shibata, N. Molecules 2019, 24, 221.

[37] Jiang, L. Q.; Yi, W. B.; Liu, Q. R. Adv. Synth. Catal. 2016, 358, 3700 .

[38] Zhao, X.; Wei, A. Q.; Li, T. J.; Su, Z. Y.; Chen, J.; Lu, K. Org. Chem. Front. 2017, 4, 232.

[39] Zhao, X.; Li, T. J.; Yang, B.; Qiu, D.; Lu, K. Tetrahedron 2017, 73, 3112 .

[40] Jiang, L. Q.; Ding, T. Q.; Yi, W. B.; Zhang, W. Org. Lett. 2018, 20, 2236 .

[41] Jiang, L. Q.; Yan, Q.; Wang, R. K.; Ding, T. Q.; Yi, W. B.; Zhang, W. Chem.-Eur. J. 2018, 24, 18749.

[42] Yan, Q.; Jiang, L. Q.; Yi, W. B.; Liu, Q. R.; Zhang, W. Adv. Synth. Catal. 2017, 359, 2471.

[43] Huang, Z. Y.; Matsubara, O.; Jia, S. C.; Tokunag, E.; Shibata, N. Org. Lett. 2017, 19, 934.

[44] Zhang, P. P.; Lü, L.; Shen, Q. L. Acta Chim. Sinica 2017, 75, 744 (in Chinese). (张盼盼, 吕龙, 沈其龙, 化学学报, 2017, 75, 744.)

[45] Hu, J. J.; Huang, Y. G.; Xu, X. H.; Qing, F. L. Chin. J. Org. Chem. 2018, 38, 177 (in Chinese). (胡娟娟, 黄焰根, 徐修华, 卿凤翎, 有机化学, 2018, 38, 177.)

[46] Wu, J.; Gu, Y.; Leng, X. B.; Shen, Q. L. Angew. Chem., Int. Ed. 2015, 54, 7648.

[47] Wu, J.; Liu, Y. F.; Lu, C. H.; Shen, Q. L. Chem. Sci. 2016, 7, 3757.

[48] Wu, J.; Lu, C. H.; Lu, L.; Shen, Q. L. Chin. J. Chem. 2018, 36, 1031.

[49] Zhu, D. H.; Shao, X. X.; Hong, X.; Lu, L.; Shen, Q. L. Angew. Chem., Int. Ed. 2016, 55, 15807.

[50] Xu, B.; Wang, D. C.; Hu, Y. H.; Shen, Q. L. Org. Chem. Front. 2018, 5, 1462 .

[51] Li, J. B.; Zhu, D. H.; Lv, L. Y.; Li, C. J. Chem. Sci. 2018, 9, 5781.

[52] Guo, S. H.; Zhang, X. L.; Pan, G. F.; Zhu, X. Q.; Gao, Y. R.; Wang, Y. Q. Angew. Chem., Int. Ed. 2018, 57, 1663.

[53] Li, H. Y.; Cheng, Z. R.; Tung, C. H.; Xu, Z. H. ACS Catal. 2018, 8, 
8237.

[54] Xuan, J.; Zhang, Z. G.; Xiao, W. J. Angew. Chem., Int. Ed. 2015, $54,15632$.

[55] Prier, C. K.; Rankic, D. A.; MacMillan, W. C. Chem. Rev. 2013, 113, 5322.

[56] Candish, L.; Pitzer, L.; Gomez, S. A.; Glorius. F. Chem.-Eur. J. 2016, 22, 4753 .

[57] Xu, W. T.; Ma, J. Y.; Yuan, X. A.; Dai, J.; Xie, J.; Zhu, C. J. Angew. Chem., Int. Ed. 2018, 57, 10357.

[58] Jouvin, K.; Matheis, C.; Goossen, L. J. Chem.-Eur. J. 2015, 21, 14324.

[59] Ding, T. Q.; Jiang, L. Q.; Yi, W. B. Org. Lett. 2018, 20, 170.

[60] Yu, J.; Lin, J. H.; Xiao, J. C. Angew. Chem., Int. Ed. 2017, 56, 16669.

[61] Yang, O.; Gooßen, L. J. Asian J. Org. Chem. 2018, 8, 650.

[62] Xu, L. J.; Wang, H. Y.; Zheng, C. W.; Zhao, G. Tetrahedron 2017, $73,6057$.

[63] Shen, F.; Zhang, P. P.; Lu, L.; Shen, Q. L. Org. Lett. 2017, 19, 1032.

[64] Ismalaj, E.; Bars, D. L.; Billard T. Angew. Chem., Int. Ed. 2016, 55, 4790.

[65] Ismalaj, E.; Billard, T. J. Fluorine Chem. 2017, 203, 215.

[66] Ismalaj, E.; Glenadel, Q.; Billard T. Eur. J. Org. Chem. 2017, 14, 1911.

[67] Xiong, H. Y.; Bayle, A.; Pannecoucke, X.; Besset, T. Angew. Chem., Int. Ed. 2016, 55, 13490.

[68] Robins, M. J.; Wnuk, S. F. J. Org. Chem. 1993, 58, 3800

[69] Shinichi, A.; Norihiko, Y.; Tsuyoshi, F.; Shoji, H. Bull. Chem. Soc. Jpn. 2002, 75, 1597.

[70] Lal, G. S.; Pez, G. P.; Pesaresi, R. J.; Prozonic, F. M.; Cheng, H. J. Org. Chem. 1999, 64, 7048.

[71] Matthews, D. P.; Persichetti, R. A.; McCarthy, J. R. Org. Prep. Proced. Int. 1994, 26, 605.

[72] Phillipps, G. H.; Bailey, E. J.; Bain, B. M.; Borella. R. A.; Buckton,
J. B.; Clark, J. C.; Doherty, A. E.; English, A. F.; Fazakerley, H.; Laing, S. B.; Allman, E. L.; Robinson, J. D.; Sandford, P. E.; Sharratt, P. J.; Steeples, I. P.; Stonehouse, R. D.; Williamson, C. J. Med. Chem. 1994, 37, 3717.

[73] Aigbirhio, F. I.; Carr, R. M.; Pike, V. W.; Steel, C. J.; Sutherland, D. R. J. Labelled Compd. Radiopharm. 1997, 39, 567.

[74] Tanigawa, M.; Kuriyama, Y.; Inagi, S.; Fuchigami, T. Electrochim. Acta 2016, 199, 314.

[75] Boys, M. L.; Collington, E. W.; Finch, H.; Swanson, S.; Whitehead, J. F. Tetrahedron Lett. 1988, 29, 3365.

[76] McCarthy, J. R.; Peet, N. P.; LeTourneau, M. E.; Inbasekaran, M. J. Am. Chem. Soc. 1985, 107, 735.

[77] Geng, Y.; Liang, A. P.; Gao, X. Y.; Niu, C. S.; Li, J. Y.; Zou, D. P.; Wu, Y. S.; Wu, Y. J. J. Org. Chem. 2017, 82, 8604.

[78] Biggadike, K.; Bledsoe, R. K.; Hassell, A. M.; Kirk, B. E.; McLay, I. M.; Shewchuk, L. M.; Stewart, E. L. J. Med. Chem. 2008, 51, 3349.

[79] Zhang, W.; Zhu, L. G.; Hu, J. B. Tetrahedron 2007, 63, 10569.

[80] Prakash, G. K. S.; Ledneczki, I.; Chacko, S.; Olah, G. A. Org. Lett. 2008, 10,557 .

[81] Zhang, M. R.; Ogawa, M.; Furutsuka, K.; Yoshida, Y.; Suzuki, K. J. Fluorine Chem. 2004, 125, 1879.

[82] Boddy, I. K.; Briggs, G. G.; Harrison, R. P.; Jones, T. H.; O'Mahony, M. J.; Marlow, I. D.; Roberts, B. G.; Willis, R. J.; Bardsley, R.; Reid, R. Pestic. Sci. 1996, 48, 189.

[83] Machara, N. P.; Ault, B. S. J. Mol. Struct. 1988, 172, 129.

[84] Zhao, Q. C.; Lu, L.; Shen, Q. L. Angew. Chem., Int. Ed. 2017, 56, 11575 .

[85] Guo, S. H.; Wang, M. Y.; Pan, G. F.; Zhu, X. Q.; Gao, Y. R.; Wang, Y. Q. Adv. Synth. Catal. 2018, 360, 1861 .

[86] Liu, F. M.; Jiang, L. Q.; Qiu, H. Y.; Yi, W. B. Org. Lett. 2018, 20, 6270 .

(Zhao, C.) 